\title{
Chiral transport in strong fields from holography
}

\author{
Yanyan Bu, ${ }^{a}$ Tuna Demircik ${ }^{b}$ and Michael Lublinsky ${ }^{b}$ \\ ${ }^{a}$ Department of Physics, Harbin Institute of Technology, \\ Harbin 150001, China \\ ${ }^{b}$ Department of Physics, Ben-Gurion University of the Negev, \\ Beer-Sheva 84105, Israel \\ E-mail: yybu@hit.edu.cn, demircik@post.bgu.ac.il, lublinm@bgu.ac.il
}

ABSTRACT: Anomaly-induced transport phenomena in presence of strong external electromagnetic fields are explored within a $4 \mathrm{D}$ field theory defined holographically as $\mathrm{U}(1)_{V} \times$ $\mathrm{U}(1)_{A}$ Maxwell-Chern-Simons theory in Schwarzschild- $A d S_{5}$. Two complementary studies are reported. In the first one, we present results on the Ohmic conductivity, diffusion constant, chiral magnetic conductivity, and additional anomaly-induced transport coefficients as functions of external e/m fields. Next, gradient resummation in a constant background magnetic field is performed. All-order resummed constitutive relations are parameterised by four momenta-dependent transport coefficient functions (TCFs). A highlight of this part is a thorough study of non-dissipative chiral magnetic waves (CMW) in strong magnetic fields.

Keywords: AdS-CFT Correspondence, Gauge-gravity correspondence, Holography and condensed matter physics (AdS/CMT), Holography and quark-gluon plasmas

ArXiv EPRINT: 1903.00896 


\section{Contents}

1 Introduction and summary 1

1.1 Motivation 1

1.2 Summary of the results: part I 3

1.3 Summary of the results: part II 5

2 Holographic setup: $\mathrm{U}(1)_{V} \times \mathrm{U}(1)_{A} \quad 6$

3 Part I: gradient expansion in external e/m fields $\quad 8$

3.1 Constitutive relations at zeroth order 8

3.2 Constitutive relations at first order $-\overrightarrow{\mathbf{B}} \neq 0, \overrightarrow{\mathbf{E}}=0 \quad 12$

3.3 Constitutive relations at first order $-\overrightarrow{\mathbf{E}} \neq 0, \overrightarrow{\mathbf{B}}=0 \quad 14$

4 Part II: gradient resummation in external magnetic field $\mathbf{1 5}$

$\begin{array}{lll}4.1 & \text { Derivation of the all-order resummed constitutive relations } & 15\end{array}$

$\begin{array}{lll}4.2 \text { The TCFs } & 18\end{array}$

$\begin{array}{lll}4.3 & \text { Non-dissipative CMW modes } & 21\end{array}$

5 Concluding remarks $\quad 23$

\section{Introduction and summary}

\subsection{Motivation}

We continue exploring transport phenomena induced by chiral anomaly in a chiral plasma with both left- and right-handed U(1) (electrically) charged fermions. The microscopic theory is defined holographically: $\mathrm{U}(1)_{V} \times \mathrm{U}(1)_{A}$ Maxwell-Chern-Simons theory in Schwarzschild- $A d S_{5}[1,2]$ to be introduced in section 2. Transport phenomena for this theory have already been extensively studied by us [3-6], and by other authors [1, 2, 7]. Here we primarily focus on some new results related to strong external e/m fields $(\vec{E}$ and $\vec{B})$.

Realistic plasmas such as quark-gluon plasma produced in heavy ion collisions or primordial plasma in the early universe are exposed to strong external e/m fields. Furthermore, the dynamics of these plasmas is governed by magneto-hydrodynamics (MHD) which generates the fields dynamically, frequently resulting in even stronger fields [8-13]. Chiral anomaly is known to modify the MHD equations, turning them into chiral MHD. An essential ingredient of any hydrodynamics is constitutive relations describing plasma medium 
effects. The constitutive relations for the vector current $J^{\mu}$ and axial current $J_{5}^{\mu}$ are of the form

$$
\vec{J}=\vec{J}\left[\rho, \rho_{5}, T, \vec{E}, \vec{B}\right] ; \quad \vec{J}_{5}=\vec{J}_{5}\left[\rho, \rho_{5}, T, \vec{E}, \vec{B}\right],
$$

where $\rho, \rho_{5}$ are vector and axial charge densities, and $T$ is temperature. The dynamics of the plasma is governed by the "conservation laws" (continuity equations)

$$
\partial_{\mu} J^{\mu}=0, \quad \partial_{\mu} J_{5}^{\mu}=12 \kappa \vec{E} \cdot \vec{B} .
$$

Note that as a result of the chiral anomaly, the global $\mathrm{U}(1)_{A}$ current is no longer conserved. $\kappa$ is the chiral anomaly coefficient $\left(\kappa=e N_{c} /\left(24 \pi^{2}\right)\right.$ for $\mathrm{SU}\left(N_{c}\right)$ gauge theory with a massless Dirac fermion in the fundamental representation and $e$ is the electric charge).

The constitutive relations (1.1) should be derived from the underlying microscopic theory. Yet, it is almost never feasible, even approximately. A great deal of modelling is inevitably employed in practice, frequently based on (truncated) gradient expansion and/or weak field approximations. Both approximations, and especially the latter one, can be inadequate. This can happen in an experimental setup, say, in chiral materials such as Weyl semimetals, in which e/m fields $\vec{E}$ and $\vec{B}$ can be controlled externally. Alternatively, plasma instabilities could generate strong fields dynamically and thus drive the system outside the applicability range of the constitutive relations. In all such cases the constitutive relations must be revised. The necessity to properly define chiral MHD in presence of strong external $\mathrm{e} / \mathrm{m}$ fields motivates our study.

In the hydrodynamic limit, the gradient expansion at each order is fixed by thermodynamics and symmetries, up to a finite number of transport coefficients (TCs). Diffusion constant, DC conductivity and shear viscosity are the most familiar examples of the lowest order TCs. However, "naive" truncation of the gradient expansion explicitly breaks relativistic invariance and thus leads to serious conceptual problems such as causality violation. Beyond conceptual issues, truncation of the gradient expansion results in numerical instabilities rendering the entire framework unreliable. Causality is restored when all order gradient terms are included, in a way providing a UV completion to the "old" hydrodynamic effective theory. The resummation generalises the concept of TC to transport coefficient functions (TCFs), which are functionals of $\partial_{t}$ and $\vec{\nabla}^{2}$ (or equivalently functions of frequency $\omega$ and three-momentum squared $\vec{q}^{2}$ in Fourier space). Therefore, TCFs contain information about infinitely many gradients and they extend the applicability of the effective theory beyond hydrodynamic limit of small frequency and momenta. When inverse Fourier transformed, the TCFs correspond to memory functions [14, 15]. Below, an effective theory based on TCFs will be referred to as the all order resummed hydrodynamics $[14,16-20]$.

The goal of the present work is to explore the generic structure of the currents (1.1), beyond the weak field limit explored by us in [3-6]. Our study will be split into two complementary directions. In the first one, we compute various TCs as functions of constant e/m fields, denoted as $\mathbf{E}, \overrightarrow{\mathbf{B}}$, up to first order in the gradient expansion. In the second part, we consider gradient resummation in the presence of a constant external magnetic field $\overrightarrow{\mathbf{B}}$ only. 


\subsection{Summary of the results: part I}

First we consider the constitutive relations (1.1) at fixed order in the gradient expansion

$$
\vec{J}=\sum_{n=0}^{\infty} \lambda^{n} \vec{J}^{[n]}, \quad \vec{J}_{5}=\sum_{n=0}^{\infty} \lambda^{n} \vec{J}_{5}^{[n]},
$$

where $\lambda$ is introduced via the replacement $\partial_{\mu} \rightarrow \lambda \partial_{\mu}$, and it counts the order in the gradient expansion.

Zeroth order $(\boldsymbol{n}=\mathbf{0})$. The most general constitutive relations are

$$
\begin{aligned}
& \vec{J}^{[0]}=\sigma_{e}^{0} \overrightarrow{\mathbf{E}}+\sigma_{\chi}^{0} \kappa \rho_{5} \overrightarrow{\mathbf{B}}+\delta \sigma_{\chi}^{0} \kappa^{2}(\overrightarrow{\mathbf{E}} \cdot \overrightarrow{\mathbf{B}}) \overrightarrow{\mathbf{B}}+\sigma_{\chi H}^{0} \kappa^{2} \rho \overrightarrow{\mathbf{B}} \times \overrightarrow{\mathbf{E}}+\sigma_{\chi e^{0}}^{0} \kappa^{3} \rho_{5}(\overrightarrow{\mathbf{B}} \cdot \overrightarrow{\mathbf{E}}) \overrightarrow{\mathbf{E}}, \\
& \vec{J}_{5}^{[0]}=\sigma_{\chi}^{0} \kappa \rho \overrightarrow{\mathbf{B}}+\sigma_{\chi H}^{0} \kappa^{2} \rho_{5} \overrightarrow{\mathbf{B}} \times \overrightarrow{\mathbf{E}}+\sigma_{\chi}^{0} \kappa^{3} \rho(\overrightarrow{\mathbf{B}} \cdot \overrightarrow{\mathbf{E}}) \overrightarrow{\mathbf{E}}+\sigma_{s}^{0} \kappa^{3}(\overrightarrow{\mathbf{E}} \cdot \overrightarrow{\mathbf{B}}) \overrightarrow{\mathbf{B}} \times \overrightarrow{\mathbf{E}} .
\end{aligned}
$$

Here one recognises some familiar terms. In the vector current: the Ohmic conductivity $(\vec{J} \sim \overrightarrow{\mathbf{E}})$, the chiral magnetic effect $(\mathrm{CME})\left(\vec{J} \sim \rho_{5} \overrightarrow{\mathbf{B}}\right)[21-23]$, the chiral Hall effect $(\vec{J} \sim \overrightarrow{\mathbf{B}} \times \overrightarrow{\mathbf{E}})[24]$. In the axial current one notices the chiral separation effect (CSE) $\left(\vec{J}_{5} \sim \rho \overrightarrow{\mathbf{B}}\right)[25,26]$ and the chiral electric separation effect (CESE) $\left(\vec{J}_{5} \sim \rho \overrightarrow{\mathbf{E}}\right)[27]$.

Apart of the last term in $\vec{J}_{5}^{[0]}$, which predicts separation of chiral charge along the Poynting vector $\overrightarrow{\mathbf{S}}=\mathbf{E} \times \overrightarrow{\mathbf{B}}$, all the terms in (1.4), (1.5) have already appeared in the literature, particularly in our previous publications $[3,5]$. The main novelty of the present work is that we consider all the TCs in (1.4), (1.5) as scalar functions of external e/m fields

$$
\sigma_{e}^{0}=\sigma_{e}^{0}\left[\mathbf{E}^{2}, \mathbf{B}^{2},(\overrightarrow{\mathbf{B}} \cdot \overrightarrow{\mathbf{E}})^{2}\right] ; \quad \sigma_{\chi}^{0}=\sigma_{\chi}^{0}\left[\mathbf{E}^{2}, \mathbf{B}^{2},(\overrightarrow{\mathbf{B}} \cdot \overrightarrow{\mathbf{E}})^{2}\right] ; \quad \text { etc },
$$

without assuming any weak field approximation, in contrast to what has been done in the past.

One might prefer an alternative representation of (1.4), (1.5) reflecting apparent anisotropy induced by the external fields.

$$
\begin{aligned}
J_{i}^{[0]}= & \sigma_{e}^{0}\left(\delta_{i j}-\frac{\mathbf{B}_{i} \mathbf{B}_{j}}{\mathbf{B}^{2}}\right) \mathbf{E}_{j}+\sigma_{e}^{0 \mathrm{~L}} \frac{\mathbf{B}_{i} \mathbf{B}_{j}}{\mathbf{B}^{2}} \mathbf{E}_{j}+\sigma_{\chi}^{0} \kappa \rho_{5}\left(\delta_{i j}-\frac{\mathbf{E}_{i} \mathbf{E}_{j}}{\mathbf{E}^{2}}\right) \mathbf{B}_{j} \\
& +\sigma_{\chi}^{0 \mathrm{~L}} \kappa \rho_{5} \frac{\mathbf{E}_{i} \mathbf{E}_{j}}{\mathbf{E}^{2}} \mathbf{B}_{j}+\sigma_{\chi H}^{0} \kappa^{2} \rho(\overrightarrow{\mathbf{B}} \times \overrightarrow{\mathbf{E}})_{i}, \\
J_{5 i}^{[0]}= & \sigma_{\chi}^{0} \kappa \rho\left(\delta_{i j}-\frac{\mathbf{E}_{i} \mathbf{E}_{j}}{\mathbf{E}^{2}}\right) \mathbf{B}_{j}+\sigma_{\chi}^{0 \mathrm{~L}} \kappa \rho \frac{\mathbf{E}_{i} \mathbf{E}_{j}}{\mathbf{E}^{2}} \mathbf{B}_{j}+\sigma_{\chi H}^{0} \kappa^{2} \rho_{5}(\overrightarrow{\mathbf{B}} \times \overrightarrow{\mathbf{E}})_{i} \\
& +\sigma_{s}^{0} \kappa^{3}(\overrightarrow{\mathbf{E}} \cdot \overrightarrow{\mathbf{B}})(\overrightarrow{\mathbf{B}} \times \overrightarrow{\mathbf{E}})_{i},
\end{aligned}
$$

with the longitudinal Ohmic and CME conductivities

$$
\sigma_{e}^{0 \mathrm{~L}}=\sigma_{e}^{0}+\kappa^{2} \mathbf{B}^{2} \delta \sigma_{\chi}^{0}, \quad \sigma_{\chi}^{0 \mathrm{~L}}=\sigma_{\chi}^{0}+\kappa^{2} \mathbf{E}^{2} \sigma_{\chi e}^{0} .
$$

The constitutive relations (1.4), (1.5) are in a sense "off-shell" since the charge densities $\rho$ and $\rho_{5}$ are treated as independent of the three-currents $\vec{J}$ and $\overrightarrow{J_{5}}$. Imposing the continuity equations (1.2), the constitutive relations (1.1) are put "on-shell". To the leading order in spatial momentum, (1.4), (1.5) result in a dispersion relation for the chiral plasma,

$$
\omega= \pm \sigma_{\chi}^{0} \kappa \vec{q} \cdot \overrightarrow{\mathbf{B}} \pm \sigma_{\chi e^{0}}^{0} \kappa^{3}(\overrightarrow{\mathbf{B}} \cdot \overrightarrow{\mathbf{E}}) \vec{q} \cdot \overrightarrow{\mathbf{E}}+\sigma_{\chi H}^{0} \kappa^{2} \vec{q} \cdot(\overrightarrow{\mathbf{B}} \times \overrightarrow{\mathbf{E}})+\mathcal{O}\left(q^{2}\right)
$$


There are three types of gapless modes propagating in the chiral plasma: the chiral magnetic wave $(\mathrm{CMW})^{1}$ [30], the chiral electric wave (CEW) [24] and the chiral Hall density wave $(\mathrm{CHDW})[5,6]$. The external field dependent TCs $\sigma_{\chi}^{0}, \sigma_{\chi e}^{0}, \sigma_{\chi H}^{0}$ reflect speeds of these modes. Note that, in contrast to CMW and CEW both propagating in two opposite directions, CHDW is directed along the Poynting vector only.

Our goal is to compute the dependence of the TCs above on the external fields. The only analytic result that has already been known in the literature is for $\sigma_{\chi}^{0}[\overrightarrow{\mathbf{E}}=0][4,31,32]$

$$
v_{\chi} \equiv \kappa \mathbf{B} \sigma_{\chi}^{0}=\frac{\Gamma\left[\left(3-\sqrt{1-144 \kappa^{2} \mathbf{B}^{2}}\right) / 4\right] \Gamma\left[\left(3+\sqrt{1-144 \kappa^{2} \mathbf{B}^{2}}\right) / 4\right]}{3 \kappa \mathbf{B} \Gamma\left[\left(1-\sqrt{1-144 \kappa^{2} \mathbf{B}^{2}}\right) / 4\right] \Gamma\left[\left(1+\sqrt{1-144 \kappa^{2} \mathbf{B}^{2}}\right) / 4\right]},
$$

where $\Gamma[x]$ is the Gamma function and all the units are rescaled by the temperature, $\pi T=1$. Small and large field limits are

$$
\begin{aligned}
\sigma_{\chi}^{0} & \rightarrow 6+216(1-2 \log 2) \kappa^{2} \mathbf{B}^{2}+\mathcal{O}\left(\mathbf{B}^{4}\right), \quad \text { as } \quad \kappa \mathbf{B} \rightarrow 0, \\
\sigma_{\chi}^{0} & \rightarrow \frac{1}{\kappa \mathbf{B}} \quad \text { as } \quad \kappa \mathbf{B} \rightarrow \infty .
\end{aligned}
$$

Thus, in the strong magnetic field limit, the speed of CMW $v_{\chi}$ goes to that of light [30].

While we were not able to obtain any new analytical insights, all the TCs in (1.4), (1.5) were computed numerically and the results are presented in section 3.1. Here we quote some asymptotic behaviours for large- $\kappa \mathbf{B}$ and large- $\kappa \mathbf{E}$

$$
\begin{array}{lc}
\delta \sigma_{\chi}^{0}[\kappa \mathbf{B} \rightarrow \infty, \kappa \mathbf{E}=0] \rightarrow \frac{3.349}{(\kappa \mathbf{B})^{3 / 2},}, & \sigma_{\chi H}^{0}[\kappa \mathbf{B} \rightarrow \infty, \kappa \mathbf{E}=0] \rightarrow-\frac{1}{(\kappa \mathbf{B})^{2}}, \\
\sigma_{\chi e}^{0}[\kappa \mathbf{B} \rightarrow \infty, \kappa \mathbf{E}=0] \rightarrow \frac{0.977}{(\kappa \mathbf{B})^{3}}, & \sigma_{s}^{0}[\kappa \mathbf{B} \rightarrow \infty, \kappa \mathbf{E}=0] \rightarrow-\frac{6.751}{(\kappa \mathbf{B})^{2}}, \\
\delta \sigma_{\chi}^{0}[\kappa \mathbf{B}=0, \kappa \mathbf{E} \rightarrow \infty] \rightarrow-\frac{2.243}{(\kappa \mathbf{E})^{3 / 2}}, & \sigma_{\chi H}^{0}[\kappa \mathbf{B}=0, \kappa \mathbf{E} \rightarrow \infty] \rightarrow-\frac{1}{(\kappa \mathbf{E})^{2}}, \\
\sigma_{\chi e}^{0}[\kappa \mathbf{B}=0, \kappa \mathbf{E} \rightarrow \infty] \rightarrow \frac{6.04}{(\kappa \mathbf{E})^{2}}, & \sigma_{s}^{0}[\kappa \mathbf{B}=0, \kappa \mathbf{E} \rightarrow \infty] \rightarrow-\frac{3.069}{(\kappa \mathbf{E})^{5 / 2}} .
\end{array}
$$

At large $\kappa \mathbf{E}, \sigma_{\chi}^{0}[\kappa \mathbf{B}=0]$ decays much faster than any other TCs, and asymptotically does not scale as a power function of $\kappa \mathbf{E}$. $\sigma_{e}^{0}$ has certain asymptotic behaviour at large values of $\mathrm{e} / \mathrm{m}$ fields only when $\kappa \mathbf{E}=\kappa \mathbf{B}$

$$
\sigma_{e}^{0}[\kappa \mathbf{B}=\kappa \mathbf{E} \rightarrow \infty] \simeq 1+3.09(\kappa \mathbf{E} \cdot \kappa \mathbf{B})^{0.27} .
$$

First order $(\boldsymbol{n}=\mathbf{1})$. We consider the cases of either $\mathbf{E}=0$ or $\mathbf{B}=0$ separately:

$$
\begin{array}{ll}
\mathbf{E}=0: & \vec{J}^{[1]}=-\mathcal{D}_{0} \vec{\nabla} \rho+\tau_{\bar{\chi}} \kappa \partial_{t} \rho_{5} \overrightarrow{\mathbf{B}}+\mathcal{D}_{B}^{0} \kappa^{2}(\overrightarrow{\mathbf{B}} \cdot \vec{\nabla} \rho) \overrightarrow{\mathbf{B}}, \\
& \vec{J}_{5}^{[1]}=-\mathcal{D}_{0} \vec{\nabla} \rho_{5}+\tau_{\bar{\chi}} \kappa \partial_{t} \rho \overrightarrow{\mathbf{B}}+\mathcal{D}_{B}^{0} \kappa^{2}\left(\overrightarrow{\mathbf{B}} \cdot \vec{\nabla} \rho_{5}\right) \overrightarrow{\mathbf{B}} . \\
\mathbf{B}=0: & \vec{J}^{[1]}=-\mathcal{D}_{0} \vec{\nabla} \rho+\sigma_{a \chi}^{0} \kappa \overrightarrow{\mathbf{E}} \times \vec{\nabla} \rho_{5}+\mathcal{D}_{E}^{0} \kappa^{2}(\overrightarrow{\mathbf{E}} \cdot \vec{\nabla} \rho) \overrightarrow{\mathbf{E}}, \\
& \vec{J}_{5}^{[1]}=-\mathcal{D}_{0} \vec{\nabla} \rho_{5}+\sigma_{a \chi}^{0} \kappa \overrightarrow{\mathbf{E}} \times \vec{\nabla} \rho+\mathcal{D}_{E}^{0} \kappa^{2}\left(\overrightarrow{\mathbf{E}} \cdot \vec{\nabla} \rho_{5}\right) \overrightarrow{\mathbf{E}} .
\end{array}
$$

\footnotetext{
${ }^{1}$ Recently, it was claimed that $[28,29]$ if the external e/m fields are promoted into dynamical, the CMW turns into a damped diffusive mode.
} 
In $[4,5]$, the diffusion constant $\mathcal{D}_{0}$ was shown to receive negative (perturbatively small) $\mathbf{E}^{2}$ - and $\mathbf{B}^{2}$-corrections induced by the chiral anomaly. Now, we are able to study this effect for arbitrary $\mathbf{E}$ and $\mathbf{B}$ fields. Particularly, we find that $\mathcal{D}_{0}$ vanishes at asymptotically large $\mathrm{e} / \mathrm{m}$ fields, see figures 5 and 6 .

The TC $\tau_{\bar{\chi}}$ is the relaxation time for CME/CSE conductivity $\sigma_{\chi}^{0}$, see (1.4), (1.5). The anomalous chiral Hall conductivity $\sigma_{a \chi H}[5,33]$ depends on the external electric field $\mathbf{E}$. $\mathcal{D}_{B}^{0}$ and $\mathcal{D}_{E}$ are new TCs. Both contribute to the longitudinal parts of the diffusion tensor, see (3.32), (3.44).

The first order TCs give rise to the decay rates of the chiral plasma modes (1.10):

$$
\begin{aligned}
& \omega= \pm \sigma_{\chi}^{0} \kappa \overrightarrow{\mathbf{B}} \cdot \vec{q}-i \mathcal{D}_{0} q^{2}-i\left(\tau_{\bar{\chi}} \sigma_{\chi}^{0}-\mathcal{D}_{B}^{0}\right) \kappa^{2}(\overrightarrow{\mathbf{B}} \cdot \vec{q})^{2}+\mathcal{O}\left(q^{3}\right), \quad \text { as } \quad \mathbf{E}=0 . \\
& \omega=-i \mathcal{D}_{0} q^{2}+i \mathcal{D}_{E}^{0} \kappa^{2}(\overrightarrow{\mathbf{E}} \cdot \vec{q})^{2}+\mathcal{O}\left(q^{3}\right), \quad \text { as } \quad \mathbf{B}=0 .
\end{aligned}
$$

When $\mathbf{B}=0$ there is no propagating mode. At $\mathbf{E}=0, \mathrm{CMW}$ is a propagating dissipative density wave. One may ask the question whether at some external magnetic field this mode becomes fully non-dissipative (real $\omega$ ). In our previous publication [6], we were able to find such a mode in a weak external magnetic field limit and for finite momenta (beyond $n=1$ approximation). It is obviously interesting to explore the effect more rigorously, and confirm the finding of [6] beyond the weak field approximation. This is one of the subjects of our second study.

All TCs in (1.16), (1.17), (1.18), (1.19) are computed numerically, see section 3.2 and 3.3. Here, we present their large- $\kappa \mathbf{B}$ and large- $\kappa \mathbf{E}$ behavior:

$$
\begin{aligned}
& \mathcal{D}_{0}[\kappa \mathbf{B} \rightarrow \infty, \kappa \mathbf{E}=0] \rightarrow \frac{0.083}{(\kappa \mathbf{B})}, \quad \tau_{\bar{\chi}}[\kappa \mathbf{B} \rightarrow \infty, \kappa \mathbf{E}=0] \rightarrow-\frac{0.36}{(\kappa \mathbf{B})^{3 / 2}}, \\
& \mathcal{D}_{B}^{0}[\kappa \mathbf{B} \rightarrow \infty, \kappa \mathbf{E}=0] \rightarrow-\frac{0.269}{(\kappa \mathbf{B})^{5 / 2} .} \\
& \sigma_{a \chi H}^{0}[\kappa \mathbf{B}=0, \kappa \mathbf{E} \rightarrow \infty] \rightarrow-\frac{0.141}{(\kappa \mathbf{E})^{2}}, \quad \mathcal{D}_{E}^{0}[\kappa \mathbf{B}=0, \kappa \mathbf{E} \rightarrow \infty] \rightarrow-\frac{0.298}{(\kappa \mathbf{E})^{2}} .
\end{aligned}
$$

Just like $\sigma_{\chi}^{0}[\kappa \mathbf{B}=0]$, at large- $\kappa \mathbf{E}, \mathcal{D}_{0}[\kappa \mathbf{B}=0]$ decays much faster than other TCs and asymptotically does not scale as a power function of $\kappa \mathbf{E}$.

\subsection{Summary of the results: part II}

In this part, we perform the gradient resummation taking into account inhomogeneity and time-dependence of the charge densities $\rho, \rho_{5}$. That is, the constitutive relations will be constructed to all orders in $\lambda$. The resummation technique was developed in [14, 16-20]. To this goal we neglect the terms nonlinear in $\rho, \rho_{5}$. Consequently, the currents are parameterised by four TCFs (only the case $\overrightarrow{\mathbf{E}}=0$ will be considered here),

$$
\begin{aligned}
\vec{J} & =-\mathcal{D} \vec{\nabla} \rho+\mathcal{D}_{B} \kappa^{2} \overrightarrow{\mathbf{B}}(\overrightarrow{\mathbf{B}} \cdot \vec{\nabla} \rho)+\bar{\sigma}_{\bar{\chi}} \kappa \overrightarrow{\mathbf{B}} \rho_{5}+\mathcal{D}_{\chi} \kappa(\overrightarrow{\mathbf{B}} \cdot \vec{\nabla}) \vec{\nabla} \rho_{5} \\
\overrightarrow{J_{5}} & =-\mathcal{D} \vec{\nabla} \rho_{5}+\mathcal{D}_{B} \kappa^{2} \overrightarrow{\mathbf{B}}\left(\overrightarrow{\mathbf{B}} \cdot \vec{\nabla} \rho_{5}\right)+\bar{\sigma}_{\bar{\chi}} \kappa \overrightarrow{\mathbf{B}} \rho+\mathcal{D}_{\chi} \kappa(\overrightarrow{\mathbf{B}} \cdot \vec{\nabla}) \vec{\nabla} \rho .
\end{aligned}
$$

The TCFs $\mathcal{D}\left[\omega, q^{2}\right], \bar{\sigma}_{\bar{\chi}}\left[\omega, q^{2}\right]$ and $\mathcal{D}_{\chi}\left[\omega, q^{2}\right]$ were introduced in $[3,6,15]$. Here they are promoted into $\overrightarrow{\mathbf{B}}$-dependent, $\mathcal{D}\left[\omega, q^{2} ; \mathbf{B}^{2},(\vec{q} \cdot \overrightarrow{\mathbf{B}})^{2}\right]$, etc. $\mathcal{D}_{B}$ is a new TCF, and the relevant 
TC appeared in [5] at finite (third) order only. In section 4, these TCFs are calculated numerically for generic values of frequency/momenta and magnetic field.

Beyond some critical value of $\kappa \mathbf{B} \gtrsim 0.5$, these TCFs display singular behavior at certain values of real $\omega$, identified with quasi-normal modes (QNMs). These QNMs become real at large $\mathbf{B}[32,34]$, and their $\mathbf{B}$ dependence is that of a Landau level. These real modes lead to a phenomenon of anomalous resonance [35].

The resummed constitutive relations (1.24), (1.25) give rise to exact dispersion relations for the CMW, beyond small frequency/momenta approximations of (1.10), (1.20), (1.21),

$$
\omega= \pm\left(\bar{\sigma}_{\bar{\chi}}-q^{2} \mathcal{D}_{\chi}\right) \kappa \overrightarrow{\mathbf{B}} \cdot \vec{q}-i\left(q^{2} \mathcal{D}-\mathcal{D}_{B}(\kappa \overrightarrow{\mathbf{B}} \cdot \vec{q})^{2}\right)
$$

Since the TCFs are complex functions of the frequency/momenta, (1.26) is expected to have infinitely many solutions, including many gapped modes. In section 4 , we will demonstrate that there are purely real, and thus non-dissipative solutions to (1.26), similar to the ones discovered by us in [6] based on weak magnetic field analysis.

The rest of this paper is structured as follows. Section 2 presents the holographic setup. Section 3 introduces calculational details for Part I and displays numerical results for the TCs mentioned above. Section 4 presents the results for Part II. Section 5 contains concluding remarks.

\section{Holographic setup: $\mathrm{U}(1)_{V} \times \mathrm{U}(1)_{A}$}

The holographic model is a Maxwell-Chern-Simons theory with two U(1) fields in the Schwarzschild- $A d S_{5}$. A more detailed presentation of the model could be found in [1-6]. The bulk action is

$$
S=\int d^{5} x \sqrt{-g} \mathcal{L}+S_{\text {c.t. }}
$$

where

$$
\begin{aligned}
\mathcal{L}= & -\frac{1}{4}\left(F^{V}\right)_{M N}\left(F^{V}\right)^{M N}-\frac{1}{4}\left(F^{a}\right)_{M N}\left(F^{a}\right)^{M N}+\frac{\kappa \epsilon^{M N P Q R}}{2 \sqrt{-g}} \\
& \times\left[3 A_{M}\left(F^{V}\right)_{N P}\left(F^{V}\right)_{Q R}+A_{M}\left(F^{a}\right)_{N P}\left(F^{a}\right)_{Q R}\right]
\end{aligned}
$$

and the counter-term action $S_{\text {c.t. }}$ is

$$
S_{\text {c.t. }}=\frac{1}{4} \log r \int d^{4} x \sqrt{-\gamma}\left[\left(F^{V}\right)_{\mu \nu}\left(F^{V}\right)^{\mu \nu}+\left(F^{a}\right)_{\mu \nu}\left(F^{a}\right)^{\mu \nu}\right] .
$$

Above, $\left(F^{V}\right)_{M N}$ and $\left(F^{a}\right)_{M N}$ denote the field strengths for the vector $V$ and axial $A$ gauge fields in the bulk, respectively. $\kappa$ is Chern-Simons coupling. The terms proportional to $\kappa$ mimic chiral anomaly of the boundary field theory.

In the ingoing Eddington-Finkelstein coordinate, the line element of the metric of Schwarzschild- $A d S_{5}$ is

$$
d s^{2}=g_{M N} d x^{M} d x^{N}=2 d t d r-r^{2} f(r) d t^{2}+r^{2} \delta_{i j} d x^{i} d x^{j},
$$


where the blackening factor is $f(r)=1-1 / r^{4}$. The Hawking temperature, identified as temperature of the boundary theory, is normalised to $\pi T=1$.

The bulk equations of motion are

$$
\begin{aligned}
& \mathrm{EV}^{M} \equiv \nabla_{N}\left(F^{V}\right)^{N M}+\frac{3 \kappa \epsilon^{M N P Q R}}{\sqrt{-g}}\left(F^{a}\right)_{N P}\left(F^{V}\right)_{Q R}=0, \\
& \mathrm{EA}^{M} \equiv \nabla_{N}\left(F^{a}\right)^{N M}+\frac{3 \kappa \epsilon^{M N P Q R}}{2 \sqrt{-g}}\left[\left(F^{V}\right)_{N P}\left(F^{V}\right)_{Q R}+\left(F^{a}\right)_{N P}\left(F^{a}\right)_{Q R}\right]=0,
\end{aligned}
$$

where the radial and boundary components of (2.5), (2.6) correspond to dynamical and constraint equations, respectively. The boundary currents read

$$
J^{\mu} \equiv \lim _{r \rightarrow \infty} \frac{\delta S}{\delta V_{\mu}}, \quad J_{5}^{\mu} \equiv \lim _{r \rightarrow \infty} \frac{\delta S}{\delta A_{\mu}} .
$$

The radial gauge $V_{r}=A_{r}=0$ is imposed. The boundary currents (2.7) can be determined by solving the dynamical equations only, leaving the constraints aside. The dynamical equations are sufficient to fix all the TCs/TCFs while the constraints translate into the continuity equations for the boundary theory currents (1.2). We use the following ansatz for the bulk gauge fields [3, 15],

$$
V_{\mu}\left(r, x_{\alpha}\right)=\mathcal{V}_{\mu}\left(x_{\alpha}\right)-\frac{\rho\left(x_{\alpha}\right)}{2 r^{2}} \delta_{\mu t}+\mathbb{V}_{\mu}\left(r, x_{\alpha}\right), \quad A_{\mu}\left(r, x_{\alpha}\right)=-\frac{\rho_{5}\left(x_{\alpha}\right)}{2 r^{2}} \delta_{\mu t}+\mathbb{A}_{\mu}\left(r, x_{\alpha}\right) .
$$

Here $\mathcal{V}_{\mu}$ is a gauge potential of the external e/m fields $\vec{E}$ and $\vec{B}$,

$$
E_{i}=\mathcal{F}_{i t}^{V}=\partial_{i} \mathcal{V}_{t}-\partial_{t} \mathcal{V}_{i}, \quad B_{i}=\frac{1}{2} \epsilon_{i j k} \mathcal{F}_{j k}^{V}=\epsilon_{i j k} \partial_{j} \mathcal{V}_{k}
$$

Both $\mathcal{V}_{\mu}$ and $\rho, \rho_{5}$ are assumed to be known functions of the boundary coordinates, while the dynamical equations of motion are solved for $\mathbb{V}_{\mu}, \mathbb{A}_{\mu}$ as functionals of $\mathcal{V}_{\mu}$ and $\rho, \rho_{5}$. Boundary conditions are classified into three types. First, $\mathbb{V}_{\mu}$ and $\mathbb{A}_{\mu}$ are regular over the domain $r \in[1, \infty)$. Second, at the boundary $r=\infty$, we require

$$
\mathbb{V}_{\mu} \rightarrow 0, \quad \mathbb{A}_{\mu} \rightarrow 0 \quad \text { as } \quad r \rightarrow \infty .
$$

Additional integration constants will be fixed by employing the Landau frame convention for the boundary currents

$$
J^{t}=\rho\left(x_{\alpha}\right), \quad J_{5}^{t}=\rho_{5}\left(x_{\alpha}\right) .
$$

According to the holographic dictionary, the boundary currents are determined in terms of near boundary $(r=\infty)$ pre-asymptotic expansion of the bulk gauge fields:

$$
J^{\mu}=\eta^{\mu \nu}\left(2 V_{\nu}^{(2)}+2 V_{\nu}^{\mathrm{L}}+\eta^{\sigma t} \partial_{\sigma} \mathcal{F}_{t \nu}^{V}\right), \quad J_{5}^{\mu}=\eta^{\mu \nu} 2 A_{\nu}^{(2)},
$$

where $4 V_{\mu}^{\mathrm{L}}=\partial^{\nu} \mathcal{F}_{\mu \nu}^{V} . V_{\mu}^{(2)}$ and $A_{\mu}^{(2)}$ are the coefficients of $r^{-2}$ terms in the near boundary expansion of the bulk vector $V_{\mu}$ and axial $A_{\mu}$ gauge fields, respectively.

Thus, our program boils down to integrating the dynamical bulk equations, from the horizon to the boundary, for the bulk gauge fields and determining their near boundary asymptotic behaviour encoded in the coefficients $V_{\mu}^{(2)}$ and $A_{\mu}^{(2)}$. This has to be done for our specific setup of constant e/m fields corresponding to linear potential $\mathcal{V}$ of arbitrary strength. 


\section{Part I: gradient expansion in external $\mathrm{e} / \mathrm{m}$ fields}

In this section we study the non-perturbative $\overrightarrow{\mathbf{E}}$-, $\overrightarrow{\mathbf{B}}$-dependencies of the TCs introduced in section 1.2, (up to first order in the gradient expansion). The background fields $\overrightarrow{\mathbf{E}}$ and $\overrightarrow{\mathbf{B}}$ are treated as zeroth order in the gradient expansion, as opposed to our previous studies $[3,6]$. Introducing $\lambda$ as a gradient expansion parameter (by $\partial_{\mu} \rightarrow \lambda \partial_{\mu}$ ), the bulk fields $\mathbb{V}_{\mu}$ and $\mathbb{A}_{\mu}$ are expandable in powers of $\lambda$,

$$
\mathbb{V}_{\mu}=\sum_{n=0}^{\infty} \lambda^{n} \mathbb{V}_{\mu}^{[n]}, \quad \quad \mathbb{A}_{\mu}=\sum_{n=0}^{\infty} \lambda^{n} \mathbb{A}_{\mu}^{[n]} .
$$

Hence the currents $\vec{J}$ and $\vec{J}_{5}$ are expanded in $\lambda$ too, see (1.3). In what follows, we compute $\vec{J}^{[0]}, \vec{J}_{5}^{[0]}, \vec{J}^{[1]}$ and $\vec{J}_{5}^{[1]}$.

\subsection{Constitutive relations at zeroth order}

The dynamical equations for $\mathbb{V}_{t}^{[0]}$ and $\mathbb{A}_{t}^{[0]}$ are

$$
\begin{aligned}
& 0=\partial_{r}\left(r^{3} \partial_{r} \mathbb{V}_{t}^{[0]}\right)+12 \kappa \partial_{r} \mathbb{A}_{k}^{[0]} \mathbf{B}_{k} \Longrightarrow r^{3} \partial_{r} \mathbb{V}_{t}^{[0]}+12 \kappa \mathbb{A}_{k}^{[0]} \mathbf{B}_{k}=0, \\
& 0=\partial_{r}\left(r^{3} \partial_{r} \mathbb{A}_{t}^{[0]}\right)+12 \kappa \partial_{r} \mathbb{V}_{k}^{[0]} \mathbf{B}_{k} \Longrightarrow r^{3} \partial_{r} \mathbb{A}_{t}^{[0]}+12 \kappa \mathbb{V}_{k}^{[0]} \mathbf{B}_{k}=0,
\end{aligned}
$$

where the Landau frame convention (2.11) has been used to fix one integration constant in both $\mathbb{V}_{t}^{[0]}$ and $\mathbb{A}_{t}^{[0]}$. The dynamical equations for the remaining components $\mathbb{V}_{i}^{[0]}$ and $\mathbb{A}_{i}^{[0]} \operatorname{read}$

$$
\begin{aligned}
0= & \left(r^{5}-r\right) \partial_{r}^{2} \mathbb{V}_{i}^{[0]}+\left(3 r^{4}+1\right) \partial_{r} \mathbb{V}_{i}^{[0]}-r^{2} \mathbf{E}_{i}+\frac{12 \kappa}{r} \mathbf{B}_{i}\left(\rho_{5}-12 \kappa \mathbb{V}_{k}^{[0]} \mathbf{B}_{k}\right) \\
& +12 \kappa r^{2} \epsilon^{i j k} \partial_{r} \mathbb{A}_{j}^{[0]} \mathbf{E}_{k}, \\
0= & \left(r^{5}-r\right) \partial_{r}^{2} \mathbb{A}_{i}^{[0]}+\left(3 r^{4}+1\right) \partial_{r} \mathbb{A}_{i}^{[0]}+\frac{12 \kappa}{r} \mathbf{B}_{i}\left(\rho-12 \kappa \mathbb{A}_{k}^{[0]} \mathbf{B}_{k}\right)+12 \kappa r^{2} \epsilon^{i j k} \partial_{r} \mathbb{V}_{j}^{[0]} \mathbf{E}_{k} .
\end{aligned}
$$

Solutions for $\mathbb{V}_{i}^{[0]}$ and $\mathbb{A}_{i}^{[0]}$ take the form:

$$
\begin{aligned}
& \mathbb{V}_{i}^{[0]}=C_{1}^{(0)} \mathbf{E}_{i}+C_{2}^{(0)} \kappa \rho_{5} \mathbf{B}_{i}+C_{3}^{(0)} \kappa^{2}(\overrightarrow{\mathbf{E}} \cdot \overrightarrow{\mathbf{B}}) \mathbf{B}_{i}+C_{4}^{(0)} \kappa^{2} \rho(\overrightarrow{\mathbf{B}} \times \overrightarrow{\mathbf{E}})_{i}+C_{5}^{(0)} \kappa^{3} \rho_{5}(\overrightarrow{\mathbf{B}} \cdot \overrightarrow{\mathbf{E}}) \mathbf{E}_{i}, \\
& \mathbb{A}_{i}^{[0]}=C_{2}^{(0)} \kappa \rho \mathbf{B}_{i}+C_{4}^{(0)} \kappa^{2} \rho_{5}(\overrightarrow{\mathbf{B}} \times \overrightarrow{\mathbf{E}})_{i}+C_{5}^{(0)} \kappa^{3} \rho(\overrightarrow{\mathbf{B}} \cdot \overrightarrow{\mathbf{E}}) \mathbf{E}_{i}+C_{6}^{(0)} \kappa^{3}(\overrightarrow{\mathbf{B}} \cdot \overrightarrow{\mathbf{E}})(\overrightarrow{\mathbf{B}} \times \overrightarrow{\mathbf{E}})_{i},
\end{aligned}
$$

where the decomposition coefficients $C_{i}^{(0)}(i=1-6)$ are functions of $r$ only, satisfying a system of coupled ordinary differential equations (ODEs). These ODEs could be grouped into two partially decoupled sub-sectors:

$$
\begin{aligned}
& \left\{C_{1}^{(0)}, C_{3}^{(0)}, C_{6}^{(0)}\right\}: \\
& 0=\left(r^{5}-r\right) \partial_{r}^{2} C_{1}^{(0)}+\left(3 r^{4}+1\right) \partial_{r} C_{1}^{(0)}-r^{2}+12 \kappa^{4} r^{2}(\overrightarrow{\mathbf{E}} \cdot \overrightarrow{\mathbf{B}})^{2} \partial_{r} C_{6}^{(0)}, \\
& 0=\left(r^{5}-r\right) \partial_{r}^{2} C_{3}^{(0)}+\left(3 r^{4}+1\right) \partial_{r} C_{3}^{(0)}-\frac{144}{r}\left(C_{1}^{(0)}+\kappa^{2} \mathbf{B}^{2} C_{3}^{(0)}\right)-12 r^{2} \kappa^{2} \mathbf{E}^{2} \partial_{r} C_{6}^{(0)}, \\
& 0=\left(r^{5}-r\right) \partial_{r}^{2} C_{6}^{(0)}+\left(3 r^{4}+1\right) \partial_{r} C_{6}^{(0)}+12 r^{2} \partial_{r} C_{3}^{(0)} .
\end{aligned}
$$




$$
\begin{aligned}
\left\{C_{2}^{(0)}, C_{4}^{(0)}, C_{5}^{(0)}\right\}: & \\
0= & \left(r^{5}-r\right) \partial_{r}^{2} C_{2}^{(0)}+\left(3 r^{4}+1\right) \partial_{r} C_{2}^{(0)}+\frac{12}{r}-\frac{144}{r}\left(\kappa^{2} \mathbf{B}^{2} C_{2}^{(0)}+\kappa^{4}(\overrightarrow{\mathbf{E}} \cdot \overrightarrow{\mathbf{B}})^{2} C_{5}^{(0)}\right) \\
& -12 r^{2} \kappa^{2} \mathbf{E}^{2} \partial_{r} C_{4}^{(0)} \\
0= & \left(r^{5}-r\right) \partial_{r}^{2} C_{4}^{(0)}+\left(3 r^{4}+1\right) \partial_{r} C_{4}^{(0)}+12 r^{2} \partial_{r} C_{2}^{(0)} \\
0= & \left(r^{5}-r\right) \partial_{r} C_{5}^{(0)}+\left(3 r^{4}+1\right) \partial_{r} C_{5}^{(0)}+12 r^{2} \partial_{r} C_{4}^{(0)}
\end{aligned}
$$

When the solutions (3.6), (3.7) are substituted into holographic expression (2.12) for the currents, one obtains the zeroth order constitutive relations (1.4), (1.5) with the TCs given by the near boundary expansion of the decomposition coefficients $C_{i}^{(0)}$

$$
\sigma_{e}^{0}=2 c_{1}^{(0)}, \quad \sigma_{\chi}^{0}=2 c_{2}^{(0)}, \quad \delta \sigma_{\chi}^{0}=2 c_{3}^{(0)}, \quad \sigma_{\chi H}^{0}=2 c_{4}^{(0)}, \quad \sigma_{\chi e}^{0}=2 c_{5}^{(0)}, \quad \sigma_{s}^{0}=2 c_{6}^{(0)} .
$$

Here $c_{i}^{(0)}$ in (3.14) are the coefficients of $1 / r^{2}$ behaviour of $C_{i}^{(0)}$ near the boundary.

When $\overrightarrow{\mathbf{E}}=0, C_{2}^{(0)}$ was found analytically in [4,31,32], yielding an analytic expression (1.11) for $\sigma_{\chi}^{0}$. We are able to compute the remaining TCs in (1.4), (1.5) numerically only.

Prior to demonstrating new results for the field dependent TCs, we quote their values at vanishing $\mathrm{e} / \mathrm{m}$ fields (see [5] and the references therein):

$$
\begin{array}{rlrl}
\sigma_{e}^{0}(\mathbf{E} & =\mathbf{B}=0)=1, & \sigma_{\chi}^{0}(\mathbf{E}=\mathbf{B}=0) & =6, \\
\delta \sigma_{\chi}^{0}(\mathbf{E} & =\mathbf{B}=0)=18(\pi-2 \log 2), & \sigma_{\chi H}^{0}(\mathbf{E}=\mathbf{B}=0)=-36 \log 2, \\
\sigma_{\chi e}^{0}(\mathbf{E} & =\mathbf{B}=0)=9 \pi^{2}, & \sigma_{s}^{0}(\mathbf{E}=\mathbf{B}=0)=12\left(\frac{3}{8} \pi^{2}-18 \mathcal{C}\right),
\end{array}
$$

where $\mathcal{C} \approx 0.915966$ is the Catalan's constant.

In figure 1, 3D plots for all the TCs in (1.4), (1.5) are shown as functions of $\kappa \mathbf{E}$ and $\kappa \mathbf{B}$, first focusing on the case of $\overrightarrow{\mathbf{E}} \| \overrightarrow{\mathbf{B}}$. Influence of the relative angle $\theta$ between $\overrightarrow{\mathbf{E}}, \overrightarrow{\mathbf{B}}$ will be discussed later (see figure 4). Figure 1a displays $\kappa \mathbf{E}$ - and $\kappa \mathbf{B}$-dependence of the Ohmic conductivity $\sigma_{e}^{0}$. In our previous publications [3-6, 15], due to weak field assumption, the Ohmic conductivity $\sigma_{e}^{0}$ did not depend on external e/m fields at all. Here, we observe that $\sigma_{e}^{0}$ gets enhancement when $\overrightarrow{\mathbf{E}} \cdot \overrightarrow{\mathbf{B}} \neq 0$. While the dependence of Ohmic conductivity on external electric field was already considered in holography [36-38], to the best of our knowledge, anomaly-induced corrections to $\sigma_{e}^{0}$ found here have not been reported before. In [36], the nonlinear conductivity emerged from a gravitational back-reaction effect, while in $[37,38]$ it emerged from a coupling between the bulk gauge fields and an additional charged scalar. Overall, the nonlinear conductivities of [36-38] decrease with strengthening of the electric field, while, as demonstrated in the present work, the anomaly-induced effect has totally opposite signature.

All the other TCs decrease dramatically with increasing $\kappa \mathbf{E}, \kappa \mathbf{B}$, and vanish asymptotically. The results are presented normalised with respect to their values at vanishing $\kappa \mathbf{E}$ and $\kappa \mathbf{B}$. The TC $\delta \sigma_{\chi}^{0}$ turns negative in a certain region of $\kappa \mathbf{E}$ and $\kappa \mathbf{B}$ (figure 1c). 


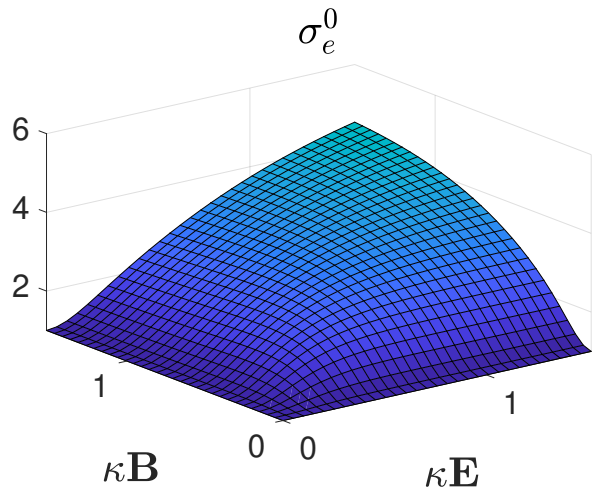

(a)

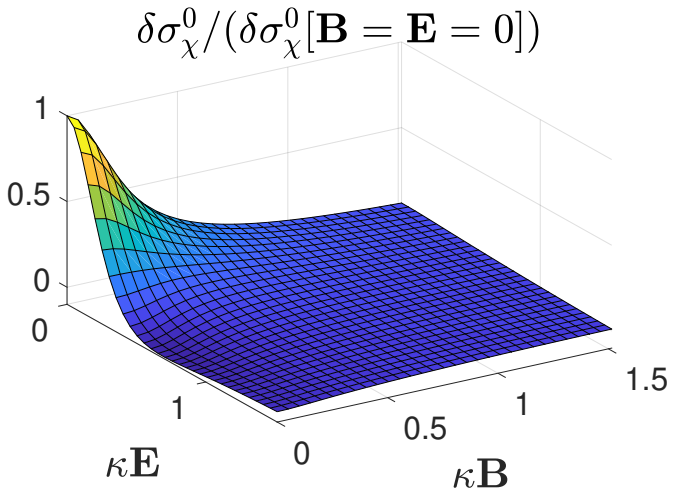

(c)

$$
\sigma_{\chi e}^{0} /\left(\sigma_{\chi e}^{0}[\mathbf{B}=\mathbf{E}=0]\right)
$$

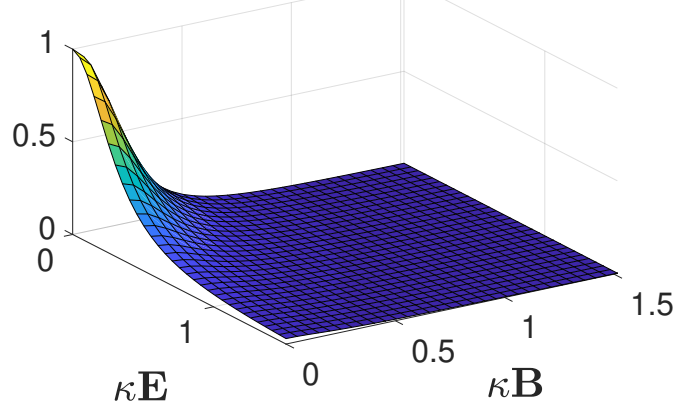

(e)

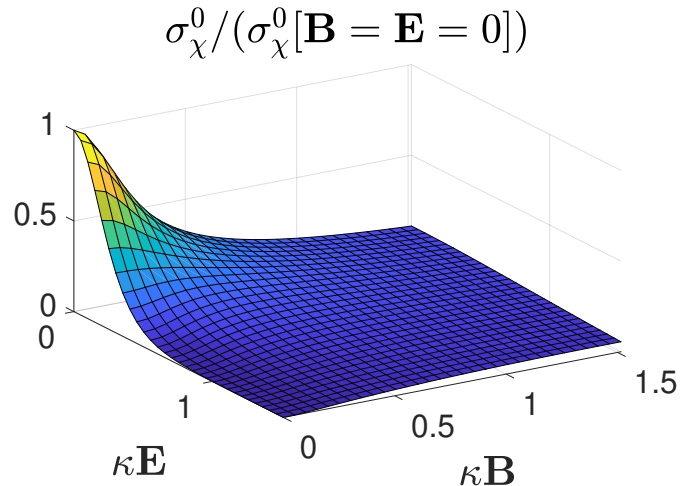

(b)

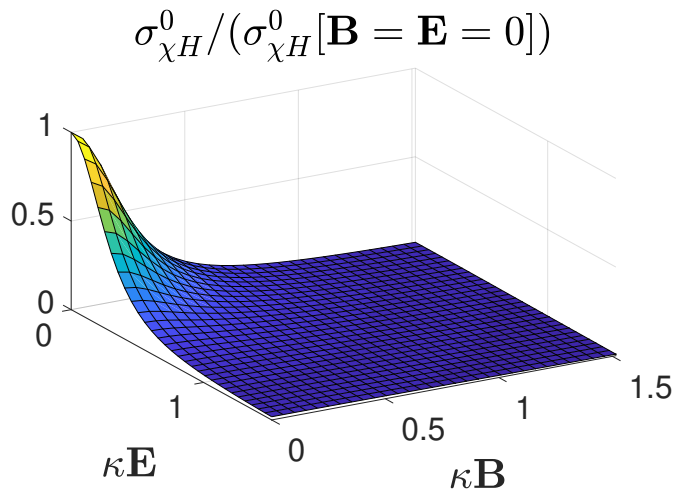

(d)

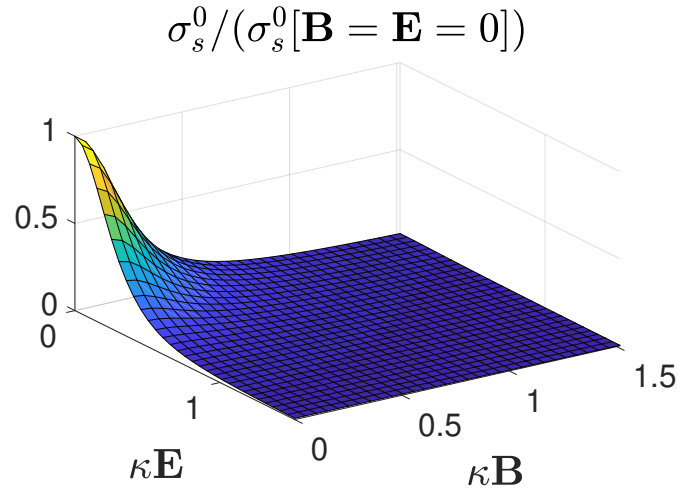

(f)

Figure 1. Zeroth order TCs as functions of $\mathbf{E}$ and $\mathbf{B}$ when $\overrightarrow{\mathbf{E}} \| \overrightarrow{\mathbf{B}}$.

Figure 2 helps to extract the asymptotic behaviour of the TCs when either $\kappa \mathbf{E}$ or $\kappa \mathbf{B}$ is very large. The TCs are rescaled so that the asymptotic scaling summarised in (1.13), (1.14) becomes transparent. Figure 3 displays rescaled $\sigma_{e}^{0}$ on $(\kappa \mathbf{E}=\kappa \mathbf{B})$-slice of figure 1a.

Finally, in figure 4 , we examine the effect of the relative angle $\theta$ between $\overrightarrow{\mathbf{E}}$ and $\overrightarrow{\mathbf{B}}$ on the TCs in (1.4), (1.5). In figure 4, the TCs are normalised with respect to their values 


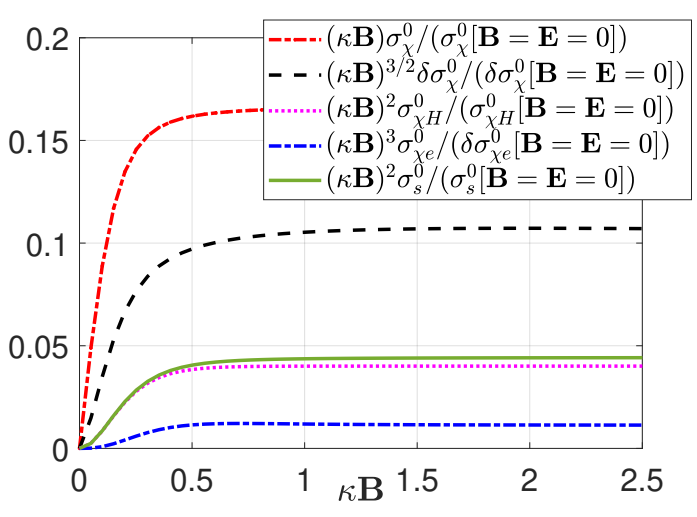

(a)

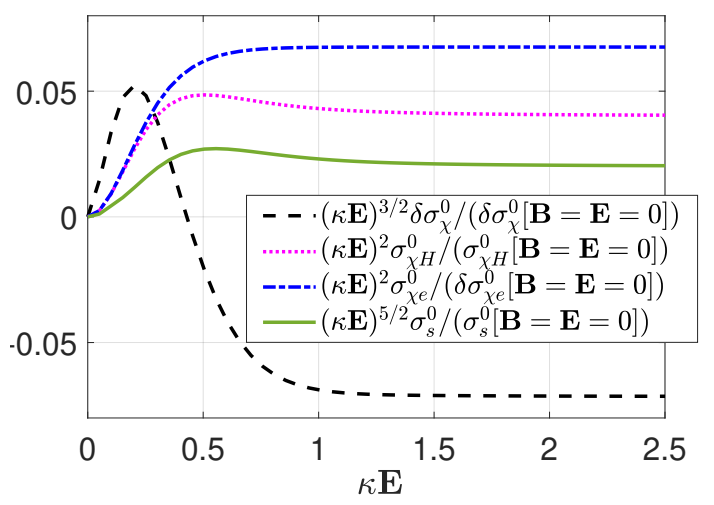

(b)

Figure 2. Normalised zeroth order TCs: (a) $\kappa \mathbf{E}=0$ and (b) $\kappa \mathbf{B}=0$.

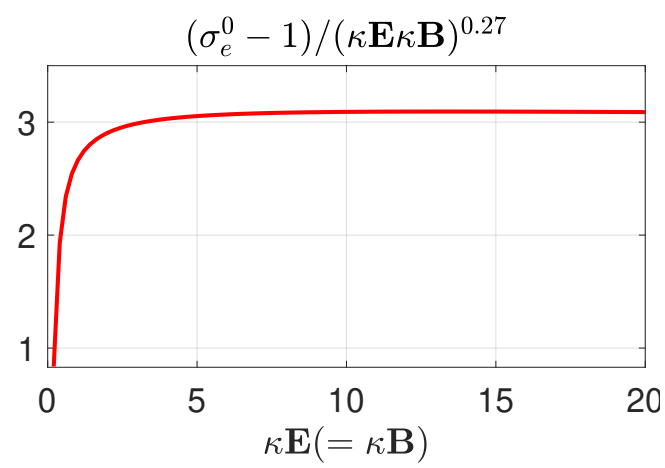

Figure 3. $\sigma_{e}^{0}$ functions of $\kappa \mathbf{B}$ and $\kappa \mathbf{E}$ (on the slice of $\kappa \mathbf{B}=\kappa \mathbf{E}$ ).

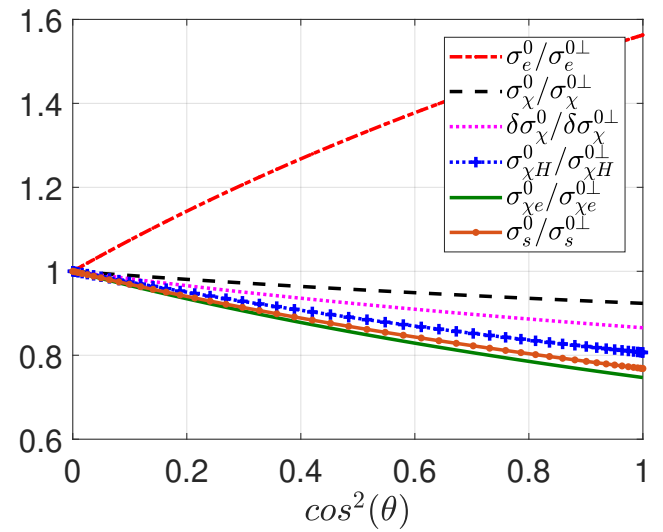

(a)

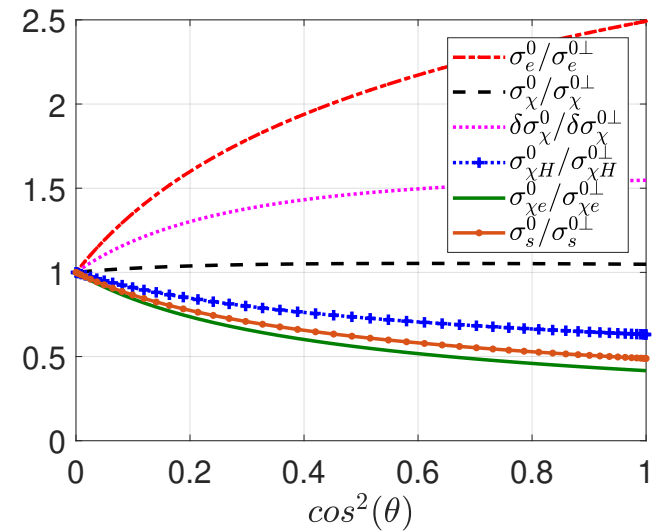

(b)

Figure 4. $\theta$-dependence of the TCs: (a) $\kappa \mathbf{B}=\kappa \mathbf{E}=0.25$ and (b) $\kappa \mathbf{B}=\kappa \mathbf{E}=0.5$. 
when $\overrightarrow{\mathbf{E}} \perp \overrightarrow{\mathbf{B}}$, denoted as $\sigma_{e}^{0 \perp}, \sigma_{\chi}^{0 \perp}, \delta \sigma_{\chi}^{0 \perp}, \sigma_{\chi H}^{0 \perp}, \sigma_{\chi e}^{0 \perp}$ and $\sigma_{s}^{0 \perp}$. Two values of the e/m fields are considered: $\kappa \mathbf{B}=\kappa \mathbf{E}=0.25$ (figure $4 \mathrm{a}$ ) and $\kappa \mathbf{B}=\kappa \mathbf{E}=0.5$ (figure $4 \mathrm{~b}$ ). We observe that the stronger the fields the more pronounced the dependence of the TCs on $\cos ^{2} \theta$. It is also very clear that while some TCs become weaker others become stronger.

Constitutive relations for $\vec{J}$ and $\vec{J}_{5}$ at first order are derived in next two subsections 3.2 and 3.3. To simplify the algebra, two cases are explored separately, either $\overrightarrow{\mathbf{E}}=0$ or $\overrightarrow{\mathbf{B}}=0$. Furthermore, only the terms linear in both $\rho$ and $\rho_{5}$ are considered. It is important to stress that smallness of the charge densities is a self-consistent approximation: when $\overrightarrow{\mathbf{E}} \cdot \overrightarrow{\mathbf{B}}=0$, there is no pumping of the axial charge into the system through the continuity equation (1.2).

3.2 Constitutive relations at first order $-\overrightarrow{\mathbf{B}} \neq 0, \overrightarrow{\mathbf{E}}=0$

When $\overrightarrow{\mathbf{E}}=0$, the dynamical equations for $\mathbb{V}_{\mu}^{[1]}$ and $\mathbb{A}_{\mu}^{[1]}$ are

$$
\begin{aligned}
0= & r^{3} \partial_{r}^{2} \mathbb{V}_{t}^{[1]}+3 r^{2} \partial_{r} \mathbb{V}_{t}^{[1]}+r \partial_{r} C_{2}^{(0)} \kappa \overrightarrow{\mathbf{B}} \cdot \vec{\nabla} \rho_{5}+12 \kappa \mathbf{B}_{k} \partial_{r} \mathbb{A}_{k}^{[1]} \\
0= & r^{3} \partial_{r}^{2} \mathbb{A}_{t}^{[1]}+3 r^{2} \partial_{r} \mathbb{A}_{t}^{[1]}+r \partial_{r} C_{2}^{(0)} \kappa \overrightarrow{\mathbf{B}} \cdot \vec{\nabla} \rho+12 \kappa \mathbf{B}_{k} \partial_{r} \mathbb{V}_{k}^{[1]} \\
0= & \left(r^{5}-r\right) \partial_{r}^{2} \mathbb{V}_{i}^{[1]}+\left(3 r^{4}+1\right) \partial_{r} \mathbb{V}_{i}^{[1]}+2 r^{3} \partial_{r} \partial_{t} \mathbb{V}_{i}^{[0]}-r^{3} \partial_{r} \partial_{i} \mathbb{V}_{t}^{[0]}+r^{2}\left(\partial_{t} \mathbb{V}_{i}^{[0]}-\partial_{i} \mathbb{V}_{t}^{[0]}\right) \\
& -\frac{1}{2} \partial_{i} \rho+12 r^{2} \kappa \mathbf{B}_{i} \partial_{r} \mathbb{A}_{t}^{[1]}, \\
0= & \left(r^{5}-r\right) \partial_{r}^{2} \mathbb{A}_{i}^{[1]}+\left(3 r^{4}+1\right) \partial_{r} \mathbb{A}_{i}^{[1]}+2 r^{3} \partial_{r} \partial_{t} \mathbb{A}_{i}^{[0]}-r^{3} \partial_{r} \partial_{i} \mathbb{A}_{t}^{[0]}+r^{2}\left(\partial_{t} \mathbb{A}_{i}^{[0]}-\partial_{i} \mathbb{A}_{t}^{[0]}\right) \\
& -\frac{1}{2} \partial_{i} \rho_{5}+12 r^{2} \kappa \mathbf{B}_{i} \partial_{r} \mathbb{V}_{t}^{[1]} .
\end{aligned}
$$

The solutions are

$$
\begin{aligned}
& \mathbb{V}_{t}^{[1]}=C_{1}^{(1)} \kappa \overrightarrow{\mathbf{B}} \cdot \vec{\nabla} \rho_{5}+C_{2}^{(1)} \partial_{t} \rho, \quad \mathbb{V}_{i}^{[1]}=C_{3}^{(1)} \partial_{i} \rho+C_{4}^{(1)} \kappa \partial_{t} \rho_{5} \mathbf{B}_{i}+C_{5}^{(1)} \kappa^{2}(\overrightarrow{\mathbf{B}} \cdot \vec{\nabla}) \rho \mathbf{B}_{i} \\
& \mathbb{A}_{t}^{[1]}=C_{1}^{(1)} \kappa \overrightarrow{\mathbf{B}} \cdot \vec{\nabla} \rho+C_{2}^{(1)} \partial_{t} \rho_{5}, \quad \mathbb{A}_{i}^{[1]}=C_{3}^{(1)} \partial_{i} \rho_{5}+C_{4}^{(1)} \kappa \partial_{t} \rho \mathbf{B}_{i}+C_{5}^{(1)} \kappa^{2}(\overrightarrow{\mathbf{B}} \cdot \vec{\nabla}) \rho_{5} \mathbf{B}_{i} .
\end{aligned}
$$

The decomposition coefficients $C_{i}^{(1)}$ obey partially decoupled ODEs, which we group into two decoupled sub-sectors:

$$
\begin{aligned}
\left\{C_{1}^{(1)}, C_{3}^{(1)}, C_{5}^{(1)}\right\} & \\
0 & =r^{3} \partial_{r}^{2} C_{1}^{(1)}+3 r^{2} \partial_{r} C_{1}^{(1)}+r \partial_{r} C_{2}^{(0)}+12\left(\partial_{r} C_{3}^{(1)}+\kappa^{2} \mathbf{B}^{2} \partial_{r} C_{5}^{(1)}\right), \\
0 & =\left(r^{5}-r\right) \partial_{r}^{2} C_{3}^{(1)}+\left(3 r^{4}+1\right) \partial_{r} C_{3}^{(1)}-r^{3} \partial_{r} F_{1}-r^{2} F_{1}-\frac{1}{2}, \\
0 & =\left(r^{5}-r\right) \partial_{r}^{2} C_{5}^{(1)}+\left(3 r^{4}+1\right) \partial_{r} C_{5}^{(1)}+12 r^{2} \partial_{r} C_{1}^{(1)},
\end{aligned}
$$

where $F_{1}$ parameterises solutions to $V_{t}^{[0]}, A_{t}^{[0]}$ (see $(3.2),(3.3)$ ):

$$
r^{3} \partial_{r} F_{1}+12 \kappa^{2} \mathbf{B}^{2} C_{2}^{(0)}=0, \quad F_{1}(r \rightarrow \infty)=0 .
$$




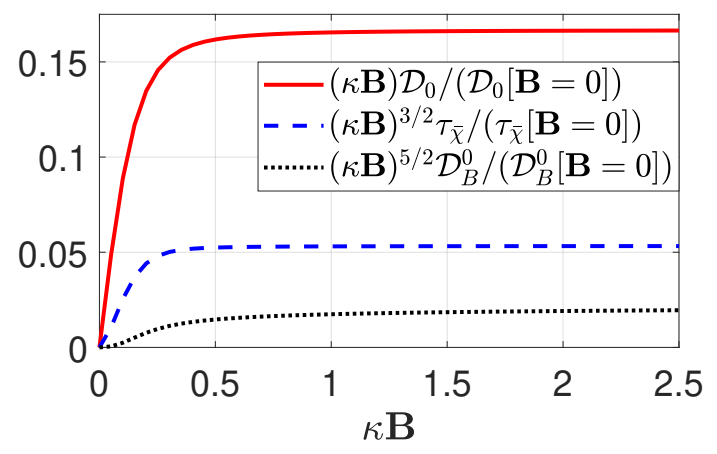

Figure 5. Normalised first order TCs as functions of $\kappa \mathbf{B}$ when $\kappa \mathbf{E}=0$.

$$
\begin{aligned}
& \left\{C_{2}^{(1)}, C_{4}^{(1)}\right\} \\
& 0=r^{3} \partial_{r}^{2} C_{2}^{(1)}+3 r^{2} \partial_{r} C_{2}^{(1)}+12 \kappa^{2} \mathbf{B}^{2} \partial_{r} C_{4}^{(1)}, \\
& 0=\left(r^{5}-r\right) \partial_{r}^{2} C_{4}^{(1)}+\left(3 r^{4}+1\right) \partial_{r} C_{4}^{(1)}+2 r^{3} \partial_{r} C_{2}^{(0)}+r^{2} C_{2}^{(0)}+12 r^{2} \partial_{r} C_{2}^{(1)} \text {. }
\end{aligned}
$$

Near the boundary $(r=\infty)$, the asymptotic expansion of the decomposition coefficients is

$$
C_{i}^{(1)}=\frac{c_{i}^{(1)}}{r^{2}}+\mathcal{O}\left(\frac{1}{r^{3}}\right), \quad i=1,2, \cdots, 5 .
$$

Plugging (3.28) into (2.12), the first order constitutive relations (1.16), (1.17) are obtained with the TCs related to the boundary data as

$$
\mathcal{D}_{0}=-2 c_{3}^{(1)}, \quad \tau_{\bar{\chi}}=2 c_{4}^{(1)}, \quad \mathcal{D}_{B}^{0}=2 c_{5}^{(1)} .
$$

Alternatively, (1.16), (1.17) can be re-expressed by collecting terms involving $\vec{\nabla} \rho, \vec{\nabla} \rho_{5}$ into transverse and longitudinal parts (with respect to $\overrightarrow{\mathbf{B}}$ ):

$$
\begin{aligned}
& J_{i}^{[1]}=-\mathcal{D}_{0}^{\mathrm{T}}\left(\delta_{i j}-\frac{\mathbf{B}_{i} \mathbf{B}_{j}}{\mathbf{B}^{2}}\right) \partial_{j} \rho-\mathcal{D}_{0}^{\mathrm{L}} \frac{\mathbf{B}_{i} \mathbf{B}_{j}}{\mathbf{B}^{2}} \partial_{j} \rho+\tau_{\bar{\chi}} \kappa \mathbf{B}_{i} \partial_{t} \rho_{5}, \\
& J_{5 i}^{[1]}=-\mathcal{D}_{0}^{\mathrm{T}}\left(\delta_{i j}-\frac{\mathbf{B}_{i} \mathbf{B}_{j}}{\mathbf{B}^{2}}\right) \partial_{j} \rho_{5}-\mathcal{D}_{0}^{\mathrm{L}} \frac{\mathbf{B}_{i} \mathbf{B}_{j}}{\mathbf{B}^{2}} \partial_{j} \rho_{5}+\tau_{\bar{\chi}} \kappa \mathbf{B}_{i} \partial_{t} \rho,
\end{aligned}
$$

where

$$
\mathcal{D}_{0}^{\mathrm{T}}=\mathcal{D}_{0}, \quad \mathcal{D}_{0}^{\mathrm{L}}=\mathcal{D}_{0}-\kappa^{2} \mathbf{B}^{2} \mathcal{D}_{B}^{0} .
$$

For generic values of $\kappa \mathbf{B}$, we are able to solve the ODEs (3.22)-(3.27) numerically only. The results are presented in figure 5 . The TCs are normalised to their values at vanishing $\mathrm{e} / \mathrm{m}$ fields (see [5]) quoted below and also rescaled by their asymptotic behaviour:

$$
\mathcal{D}_{0}[\mathbf{B}=0]=\frac{1}{2}, \quad \tau_{\bar{\chi}}[\mathbf{B}=0]=-\frac{3}{2}(\pi+2 \log 2), \quad \mathcal{D}_{B}^{0}[\mathbf{B}=0]=-9(\pi-2 \log 2) .
$$

Obviously, all the TCs in (1.16), (1.17) decrease when $\kappa \mathbf{B}$ becomes stronger, and vanish asymptotically. Large- $\kappa \mathbf{B}$ asymptotic behavior for all the TCs in (1.16), (1.17) is summarised in (1.22). 


\subsection{Constitutive relations at first order $-\overrightarrow{\mathbf{E}} \neq 0, \overrightarrow{\mathbf{B}}=0$}

The equations for $\mathbb{V}_{t}^{[1]}$ and $\mathbb{A}_{t}^{[1]}$ are homogeneous, so both $\mathbb{V}_{t}^{[1]}$ and $\mathbb{A}_{t}^{[1]}$ vanish. Consequently, the equations for $\mathbb{V}_{i}^{[1]}$ and $\mathbb{A}_{i}^{[1]}$ are

$$
\begin{aligned}
& 0=\left(r^{5}-r\right) \partial_{r}^{2} \mathbb{V}_{i}^{[1]}+\left(3 r^{4}+1\right) \partial_{r} \mathbb{V}_{i}^{[1]}-\frac{1}{2} \partial_{i} \rho+12 \kappa r^{2} \epsilon^{i j k} \partial_{r} \mathbb{A}_{j}^{[1]} \mathbf{E}_{k}-6 \kappa \epsilon^{i j k} \partial_{r} \mathbb{V}_{j}^{[0]} \partial_{k} \rho_{5}, \\
& 0=\left(r^{5}-r\right) \partial_{r}^{2} \mathbb{A}_{i}^{[1]}+\left(3 r^{4}+1\right) \partial_{r} \mathbb{A}_{i}^{[1]}-\frac{1}{2} \partial_{i} \rho_{5}+12 \kappa r^{2} \epsilon^{i j k} \partial_{r} \mathbb{V}_{j}^{[1]} \mathbf{E}_{k}-6 \kappa \epsilon^{i j k} \partial_{r} \mathbb{V}_{j}^{[0]} \partial_{k} \rho .
\end{aligned}
$$

The solutions are

$$
\begin{aligned}
& \mathbb{V}_{i}^{[1]}=C_{6}^{(1)} \partial_{i} \rho+C_{7}^{(1)} \kappa\left(\overrightarrow{\mathbf{E}} \times \vec{\nabla} \rho_{5}\right)_{i}+C_{8}^{(1)} \kappa^{2}(\overrightarrow{\mathbf{E}} \cdot \vec{\nabla} \rho) \mathbf{E}_{i}, \\
& \mathbb{A}_{i}^{[1]}=C_{6}^{(1)} \partial_{i} \rho_{5}+C_{7}^{(1)} \kappa(\overrightarrow{\mathbf{E}} \times \vec{\nabla} \rho)_{i}+C_{8}^{(1)} \kappa^{2}\left(\overrightarrow{\mathbf{E}} \cdot \vec{\nabla} \rho_{5}\right) \mathbf{E}_{i},
\end{aligned}
$$

where the decomposition coefficients satisfy coupled ODEs:

$$
\begin{aligned}
& 0=\left(r^{5}-r\right) \partial_{r}^{2} C_{6}^{(1)}+\left(3 r^{4}+1\right) \partial_{r} C_{6}^{(1)}-\frac{1}{2}+12 \kappa^{2} \mathbf{E}^{2} r^{2} \partial_{r} C_{7}^{(1)}, \\
& 0=\left(r^{5}-r\right) \partial_{r}^{2} C_{7}^{(1)}+\left(3 r^{4}+1\right) \partial_{r} C_{7}^{(1)}-12 r^{2} \partial_{r} C_{6}^{(1)}-6 \partial_{r} C_{1}^{(0)}, \\
& 0=\left(r^{5}-r\right) \partial_{r}^{2} C_{8}^{(1)}+\left(3 r^{4}+1\right) \partial_{r} C_{8}^{(1)}-12 r^{2} \partial_{r} C_{7}^{(1)} .
\end{aligned}
$$

Near $r=\infty$, the asymptotic expansion for $C_{i}^{(1)}$ is,

$$
C_{i}^{(1)}=\frac{c_{i}^{(1)}}{r^{2}}+\mathcal{O}\left(\frac{1}{r^{3}}\right), \quad i=6,7,8,
$$

which via (2.12) helps to derive the first order constitutive relations (1.18), (1.19) with the TCs given by

$$
\mathcal{D}_{0}=-2 c_{6}^{(1)}, \quad \sigma_{a \chi H}^{0}=2 c_{7}^{(1)}, \quad \mathcal{D}_{E}^{0}=2 c_{8}^{(1)} .
$$

As done for (1.16), (1.17), we combine the $\vec{\nabla} \rho, \vec{\nabla} \rho_{5}$-terms in (1.18), (1.19) into longitudinal and transverse parts (with respect to $\overrightarrow{\mathbf{E}}$ ),

$$
\begin{aligned}
& J_{i}^{[1]}=-\mathcal{D}_{0}^{\mathrm{T}}\left(\delta_{i j}-\frac{\mathbf{E}_{i} \mathbf{E}_{j}}{\mathbf{E}^{2}}\right) \partial_{j} \rho-\mathcal{D}_{0}^{\mathrm{L}} \frac{\mathbf{E}_{i} \mathbf{E}_{j}}{\mathbf{E}^{2}} \partial_{j} \rho+\sigma_{a \chi H}^{0} \epsilon^{i j k} \kappa E_{j} \partial_{k} \rho_{5}, \\
& J_{5 i}^{[1]}=-\mathcal{D}_{0}^{\mathrm{T}}\left(\delta_{i j}-\frac{\mathbf{E}_{i} \mathbf{E}_{j}}{\mathbf{E}^{2}}\right) \partial_{j} \rho_{5}-\mathcal{D}_{0}^{\mathrm{L}} \frac{\mathbf{E}_{i} \mathbf{E}_{j}}{\mathbf{E}^{2}} \partial_{j} \rho_{5}+\sigma_{a \chi}^{0} \epsilon^{i j k} \kappa E_{j} \partial_{k} \rho,
\end{aligned}
$$

where

$$
\mathcal{D}_{0}^{\mathrm{T}}=\mathcal{D}_{0}, \quad \mathcal{D}_{0}^{\mathrm{L}}=\mathcal{D}_{0}-\kappa^{2} \mathbf{E}^{2} \mathcal{D}_{E}^{0},
$$

For generic value of $\kappa \mathbf{E}$, the ODEs (3.37)-(3.39) are solved numerically and the results are displayed in figure 6. Analytical results for each TCs when $\mathbf{E}=\mathbf{B}=0$ are quoted for completeness (see [5]):

$$
\sigma_{a \chi H}^{0}[\mathbf{E}=0]=-3 \log 2, \quad \mathcal{D}_{E}^{0}[\mathbf{E}=0]=-\frac{3}{4} \pi^{2} .
$$

From figure 6, one can read off the large- $\kappa \mathbf{E}$ behavior for the TCs in (1.18), (1.19), as summarised in (1.23). We note that large- $\kappa \mathbf{E}$ behavior of $\mathcal{D}_{0}$ does not scale as a power function and it decays faster. 


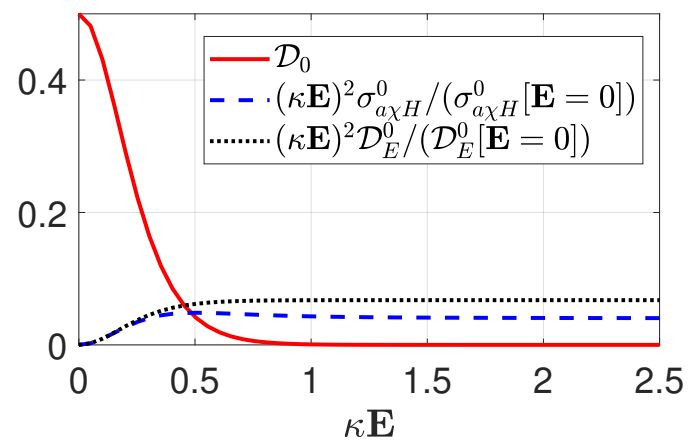

Figure 6. The first order TCs as function of $\kappa \mathbf{E}$ when $\mathbf{B}=0$.

\section{Part II: gradient resummation in external magnetic field}

In this section, we focus on all-order resummation of gradient terms that are linear in both $\rho$ and $\rho_{5}$ when $\overrightarrow{\mathbf{B}}$ is taken as arbitrary in amplitude. External electric field $\overrightarrow{\mathbf{E}}$ will be turned off throughout this section. In order to keep the terms linear in the charge densities only, we introduce a parameter $\epsilon$,

$$
\rho \rightarrow \epsilon \rho, \quad \rho_{5} \rightarrow \epsilon \rho_{5}, \quad \overrightarrow{\mathbf{B}} \sim \mathcal{O}\left(\epsilon^{0}\right) .
$$

We solve the dynamical equations up to $\mathcal{O}\left(\epsilon^{1}\right)$ :

$$
\mathbb{V}_{\mu}=\mathbb{V}_{\mu}^{(0)}+\epsilon \mathbb{V}_{\mu}^{(1)}+\cdots, \quad \mathbb{A}_{\mu}=\mathbb{A}_{\mu}^{(0)}+\epsilon \mathbb{A}_{\mu}^{(1)}+\cdots
$$

\subsection{Derivation of the all-order resummed constitutive relations}

Since the hydrodynamic variables $\rho, \rho_{5}$ are $\mathcal{O}\left(\epsilon^{1}\right)$, the zeroth order corrections $\mathbb{V}_{\mu}^{(0)}$ and $\mathbb{A}_{\mu}^{(0)}$ can depend on $\overrightarrow{\mathbf{B}}$ only and are independent of the boundary coordinates $x^{\alpha}$. Therefore, at $\mathcal{O}\left(\epsilon^{0}\right)$ the dynamical equations are

$$
\begin{aligned}
& 0=r^{3} \partial_{r}^{2} \mathbb{V}_{t}^{(0)}+3 r^{2} \partial_{r} \mathbb{V}_{t}^{(0)}+12 \kappa \mathbf{B}_{k} \partial_{r} \mathbb{A}_{k}^{(0)}, \\
& 0=\left(r^{5}-r\right) \partial_{r}^{2} \mathbb{V}_{i}^{(0)}+\left(3 r^{4}+1\right) \partial_{r} \mathbb{V}_{i}^{(0)}+12 \kappa r^{2} \mathbf{B}_{i} \partial_{r} \mathbb{A}_{t}^{(0)}, \\
& 0=r^{3} \partial_{r}^{2} \mathbb{A}_{t}^{(0)}+3 r^{2} \partial_{r} \mathbb{A}_{t}^{(0)}+12 \kappa \mathbf{B}_{k} \partial_{r} \mathbb{V}_{k}^{(0)}, \\
& 0=\left(r^{5}-r\right) \partial_{r}^{2} \mathbb{A}_{i}^{(0)}+\left(3 r^{4}+1\right) \partial_{r} \mathbb{A}_{i}^{(0)}+12 \kappa r^{2} \mathbf{B}_{i} \partial_{r} \mathbb{V}_{t}^{(0)}
\end{aligned}
$$

The equations (4.3), (4.5) could be integrated over $r$ :

$$
r^{3} \partial_{r} \mathbb{V}_{t}^{(0)}+12 \kappa \mathbf{B}_{k} \mathbb{A}_{k}^{(0)}=0, \quad \quad r^{3} \partial_{r} \mathbb{A}_{t}^{(0)}+12 \kappa \mathbf{B}_{k} \mathbb{V}_{k}^{(0)}=0,
$$

where the Landau frame convention (2.11) has been used to fix the integration constants. Hence, the equations (4.4), (4.6) become homogeneous ODEs. Combined with the boundary conditions specified in section 2, the equations (4.4), (4.6) do not have any nontrivial solution. Thus, we conclude that

$$
\mathbb{V}_{\mu}^{(0)}=\mathbb{A}_{\mu}^{(0)}=0
$$


At $\mathcal{O}\left(\epsilon^{1}\right)$, the dynamical equations become

$$
\begin{aligned}
0= & r^{3} \partial_{r}^{2} \mathbb{V}_{t}^{(1)}+3 r^{2} \partial_{r} \mathbb{V}_{t}^{(1)}+r \partial_{r} \partial_{k} \mathbb{V}_{k}^{(1)}+12 \kappa \mathbf{B}_{k} \partial_{r} \mathbb{A}_{k}^{(1)} \\
0= & \left(r^{5}-r\right) \partial_{r}^{2} \mathbb{V}_{i}^{(1)}+\left(3 r^{4}+1\right) \partial_{r} \mathbb{V}_{i}^{(1)}+2 r^{3} \partial_{r} \partial_{t} \mathbb{V}_{i}^{(1)}-r^{3} \partial_{r} \partial_{i} \mathbb{V}_{t}^{(1)}+r^{2}\left(\partial_{t} \mathbb{V}_{i}^{(1)}-\partial_{i} \mathbb{V}_{t}^{(1)}\right) \\
& +r\left(\partial^{2} \mathbb{V}_{i}^{(1)}-\partial_{i} \partial_{k} \mathbb{V}_{k}^{(1)}\right)-\frac{1}{2} \partial_{i} \rho+12 \kappa r^{2} \mathbf{B}_{i}\left(\frac{1}{r^{3}} \rho_{5}+\partial_{r} \mathbb{A}_{t}^{(1)}\right) \\
0= & r^{3} \partial_{r}^{2} \mathbb{A}_{t}^{(1)}+3 r^{2} \partial_{r} \mathbb{A}_{t}^{(1)}+r \partial_{r} \partial_{k} \mathbb{A}_{k}^{(1)}+12 \kappa \mathbf{B}_{k} \partial_{r} \mathbb{V}_{k}^{(1)} \\
0= & \left(r^{5}-r\right) \partial_{r}^{2} \mathbb{A}_{i}^{(1)}+\left(3 r^{4}+1\right) \partial_{r} \mathbb{A}_{i}^{(1)}+2 r^{3} \partial_{r} \partial_{t} \mathbb{A}_{i}^{(1)}-r^{3} \partial_{r} \partial_{i} \mathbb{A}_{t}^{(1)}+r^{2}\left(\partial_{t} \mathbb{A}_{i}^{(1)}-\partial_{i} \mathbb{A}_{t}^{(1)}\right) \\
& +r\left(\partial^{2} \mathbb{A}_{i}^{(1)}-\partial_{i} \partial_{k} \mathbb{A}_{k}^{(1)}\right)-\frac{1}{2} \partial_{i} \rho_{5}+12 \kappa r^{2} \mathbf{B}_{i}\left(\frac{1}{r^{3}} \rho+\partial_{r} \mathbb{V}_{t}^{(1)}\right)
\end{aligned}
$$

The corrections $\mathbb{V}_{\mu}^{(1)}$ and $\mathbb{A}_{\mu}^{(1)}$ are decomposed in terms of basic structures built from the external magnetic field $\overrightarrow{\mathbf{B}}$ and the charge densities $\rho, \rho_{5}$,

$$
\begin{aligned}
\mathbb{V}_{t}^{(1)}= & S_{1} \rho+S_{2} \kappa \mathbf{B}_{k} \partial_{k} \rho_{5}, \\
\mathbb{V}_{i}^{(1)}= & V_{1} \partial_{i} \rho+V_{2} \kappa^{2} \mathbf{B}_{i} \mathbf{B}_{k} \partial_{k} \rho+V_{3} \kappa \mathbf{B}_{i} \rho_{5}+V_{4} \kappa \mathbf{B}_{k} \partial_{i} \partial_{k} \rho_{5} \\
& +V_{5} \epsilon^{i j k} \kappa \mathbf{B}_{j} \partial_{k} \rho+V_{6} \kappa^{2} \epsilon^{i j k} \mathbf{B}_{j} \mathbf{B}_{l} \partial_{l} \partial_{k} \rho_{5}, \\
\mathbb{A}_{t}^{(1)}= & \bar{S}_{1} \rho_{5}+\bar{S}_{2} \kappa \mathbf{B}_{k} \partial_{k} \rho, \\
\mathbb{A}_{i}^{(1)}= & \bar{V}_{1} \partial_{i} \rho_{5}+\bar{V}_{2} \kappa^{2} \mathbf{B}_{i} \mathbf{B}_{k} \partial_{k} \rho_{5}+\bar{V}_{3} \kappa \mathbf{B}_{i} \rho+\bar{V}_{4} \kappa \mathbf{B}_{k} \partial_{i} \partial_{k} \rho \\
& +\bar{V}_{5} \epsilon^{i j k} \kappa \mathbf{B}_{j} \partial_{k} \rho_{5}+\bar{V}_{6} \epsilon^{i j k} \kappa^{2} \mathbf{B}_{j} \mathbf{B}_{l} \partial_{l} \partial_{k} \rho,
\end{aligned}
$$

where, in contrast to our previous publications [3,6], the decomposition coefficients $S_{i}, \bar{S}_{i}$, $V_{i}$ and $\bar{V}_{i}$ now depend on $\overrightarrow{\mathbf{B}}$ non-linearly. Particularly, they are scalar functionals of $\partial_{t}$, $\vec{\nabla}^{2}$ and $(\kappa \overrightarrow{\mathbf{B}} \cdot \vec{\nabla})^{2}$; and functions of $r$ and $(\kappa \mathbf{B})^{2}$. In Fourier space via $\left(\partial_{t}, \vec{\nabla}\right) \rightarrow(-i \omega, i \vec{q})$, they turn into scalar functions

$$
\begin{aligned}
& S_{i}\left(\partial_{t}, \vec{\nabla}^{2},(\kappa \overrightarrow{\mathbf{B}} \cdot \vec{\nabla})^{2} ; r,(\kappa \mathbf{B})^{2}\right) \rightarrow S_{i}\left(\omega, q^{2},(\kappa \overrightarrow{\mathbf{B}} \cdot \vec{q})^{2}, r,(\kappa \mathbf{B})^{2}\right), \\
& V_{i}\left(\partial_{t}, \vec{\nabla}^{2},(\kappa \overrightarrow{\mathbf{B}} \cdot \vec{\nabla})^{2} ; r,(\kappa \mathbf{B})^{2}\right) \rightarrow V_{i}\left(\omega, q^{2},(\kappa \overrightarrow{\mathbf{B}} \cdot \vec{q})^{2}, r,(\kappa \mathbf{B})^{2}\right), \\
& \bar{S}_{i}\left(\partial_{t}, \vec{\nabla}^{2},(\kappa \overrightarrow{\mathbf{B}} \cdot \vec{\nabla})^{2} ; r,(\kappa \mathbf{B})^{2}\right) \rightarrow \bar{S}_{i}\left(\omega, q^{2},(\kappa \overrightarrow{\mathbf{B}} \cdot \vec{q})^{2}, r,(\kappa \mathbf{B})^{2}\right), \\
& \bar{V}_{i}\left(\partial_{t}, \vec{\nabla}^{2},(\kappa \overrightarrow{\mathbf{B}} \cdot \vec{\nabla})^{2} ; r,(\kappa \mathbf{B})^{2}\right) \rightarrow \bar{V}_{i}\left(\omega, q^{2},(\kappa \overrightarrow{\mathbf{B}} \cdot \vec{q})^{2}, r,(\kappa \mathbf{B})^{2}\right) .
\end{aligned}
$$

Accordingly, the PDEs (4.9)-(4.12) give rise to a set of partially decoupled ODEs for the decomposition coefficients in (4.13)-(4.16), which we collect as 
(i): $\left\{S_{1}, \bar{S}_{2}, V_{1}, V_{2}, \bar{V}_{3}, \bar{V}_{4}\right\}$

$$
\begin{aligned}
0= & r^{3} \partial_{r}^{2} S_{1}+3 r^{2} \partial_{r} S_{1}-q^{2} r \partial_{r} V_{1}-(\kappa \overrightarrow{\mathbf{B}} \cdot \vec{q})^{2} r \partial_{r} V_{2}+12(\kappa \mathbf{B})^{2} \partial_{r} \bar{V}_{3}-12(\kappa \overrightarrow{\mathbf{B}} \cdot \vec{q})^{2} \partial_{r} \bar{V}_{4}, \\
0= & r^{3} \partial_{r}^{2} \bar{S}_{2}+3 r^{2} \partial_{r} \bar{S}_{2}+r \partial_{r} \bar{V}_{3}+12 \partial_{r} V_{1}+12(\kappa \mathbf{B})^{2} \partial_{r} V_{2}-q^{2} r \partial_{r} \bar{V}_{4} \\
0= & \left(r^{5}-r\right) \partial_{r}^{2} V_{1}+\left(3 r^{4}+1-2 i \omega r^{3}\right) \partial_{r} V_{1}-i \omega r^{2} V_{1}-r^{2}\left(S_{1}+r \partial_{r} S_{1}\right) \\
& +(\kappa \overrightarrow{\mathbf{B}} \cdot \vec{q})^{2} r V_{2}-1 / 2 \\
0= & \left(r^{5}-r\right) \partial_{r}^{2} V_{2}+\left(3 r^{4}+1-2 i \omega r^{3}\right) \partial_{r} V_{2}-i \omega r^{2} V_{2}-q^{2} r V_{2}+12 r^{2} \partial_{r} \bar{S}_{2} \\
0= & \left(r^{5}-r\right) \partial_{r}^{2} \bar{V}_{3}+\left(3 r^{4}+1-2 i \omega r^{3}\right) \partial_{r} \bar{V}_{3}-i \omega r^{2} \bar{V}_{3}-q^{2} r \bar{V}_{3}+12 r^{2} \partial_{r} S_{1}+12 / r \\
0= & \left(r^{5}-r\right) \partial_{r}^{2} \bar{V}_{4}+\left(3 r^{4}+1-2 i \omega r^{3}\right) \partial_{r} \bar{V}_{4}-i \omega r^{2} \bar{V}_{4}-r^{2}\left(\bar{S}_{2}+r \partial_{r} \bar{S}_{2}\right)-r \bar{V}_{3}
\end{aligned}
$$

(ii): $\left\{V_{5}\right\}$

$$
0=\left(r^{5}-r\right) \partial_{r}^{2} V_{5}+\left(3 r^{4}+1-2 i \omega r^{3}\right) \partial_{r} V_{5}-i \omega r^{2} V_{5}-q^{2} r V_{5}
$$

The sub-sector $\left\{\bar{S}_{1}, S_{2}, \bar{V}_{1}, \bar{V}_{2}, V_{3}, V_{4}\right\}$ satisfies the same equations as the sub-sector (i): $\left\{S_{1}, \bar{S}_{2}, V_{1}, V_{2}, \bar{V}_{3}, \bar{V}_{4}\right\}$. Given that they obey the same boundary conditions, we conclude that

$$
\left\{\bar{S}_{1}, S_{2}, \bar{V}_{1}, \bar{V}_{2}, V_{3}, V_{4}\right\}=\left\{S_{1}, \bar{S}_{2}, V_{1}, V_{2}, \bar{V}_{3}, \bar{V}_{4}\right\}
$$

The remaining functions $\bar{V}_{5}, V_{6}$ and $\bar{V}_{6}$ satisfy the same ODEs as $V_{5}$. Note that the ODEs for $V_{5}, V_{6}, \bar{V}_{5}, \bar{V}_{6}$ are homogeneous, they do not have any non-trivial solutions due to the regularity requirement (at $r=1$ ) and the vanishing boundary condition (at $r=\infty$ ):

$$
V_{5}=V_{6}=\bar{V}_{5}=\bar{V}_{6}=0 .
$$

Indeed, the conclusion $V_{5}=\bar{V}_{5}=0$ is in perfect agreement with $[5,6]$, where $V_{5}, \bar{V}_{5}$ were found to depend on $\rho, \rho_{5}$ nonlinearly.

Solving (4.21)-(4.26) near the boundary $r=\infty$ gives rise to the currents' constitutive relations $(1.24),(1.25)$ with the TCFs related to the near boundary expansion of $V_{i}$ 's:

$$
\mathcal{D}=-2 v_{1}, \quad \mathcal{D}_{B}=2 v_{2}, \quad \bar{\sigma}_{\bar{\chi}}=2 v_{3}, \quad \mathcal{D}_{\chi}=2 v_{4},
$$

where $v_{i}$ 's denote the coefficients of $1 / r^{2}$ in the expansion of $V_{i}$ 's. In contrast to our previous publications $[3,6]$, the TCFs $\mathcal{D}, \mathcal{D}_{B}, \bar{\sigma}_{\bar{\chi}}$ and $\mathcal{D}_{\chi}$ also depend on the external magnetic field $\overrightarrow{\mathbf{B}}$ non-perturbatively,

$$
\begin{aligned}
\mathcal{D} & =\mathcal{D}\left(\omega, q^{2},(\kappa \overrightarrow{\mathbf{B}} \cdot \vec{q})^{2} ;(\kappa \mathbf{B})^{2}\right), & \mathcal{D}_{B} & =\mathcal{D}_{B}\left(\omega, q^{2},(\kappa \overrightarrow{\mathbf{B}} \cdot \vec{q})^{2} ;(\kappa \mathbf{B})^{2}\right), \\
\bar{\sigma}_{\bar{\chi}} & =\bar{\sigma}_{\bar{\chi}}\left(\omega, q^{2},(\kappa \overrightarrow{\mathbf{B}} \cdot \vec{q})^{2} ;(\kappa \mathbf{B})^{2}\right), & & \mathcal{D}_{\chi}=\mathcal{D}_{\chi}\left(\omega, q^{2},(\kappa \overrightarrow{\mathbf{B}} \cdot \vec{q})^{2} ;(\kappa \mathbf{B})^{2}\right) .
\end{aligned}
$$

Determination of these TCFs is one of the novel results of the present work. Since the external magnetic field is constant, the TCFs in (1.24) measure a response of the chiral medium to inhomogeneity and time-dependence of the charge densities $\rho, \rho_{5}$. 


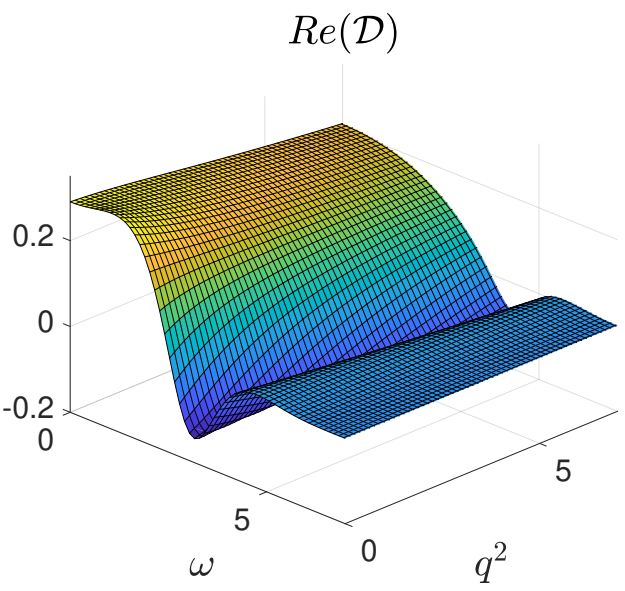

(a)

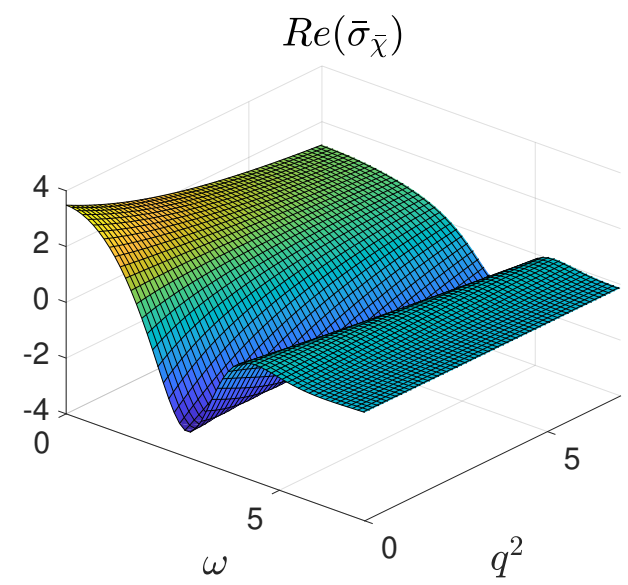

(c)

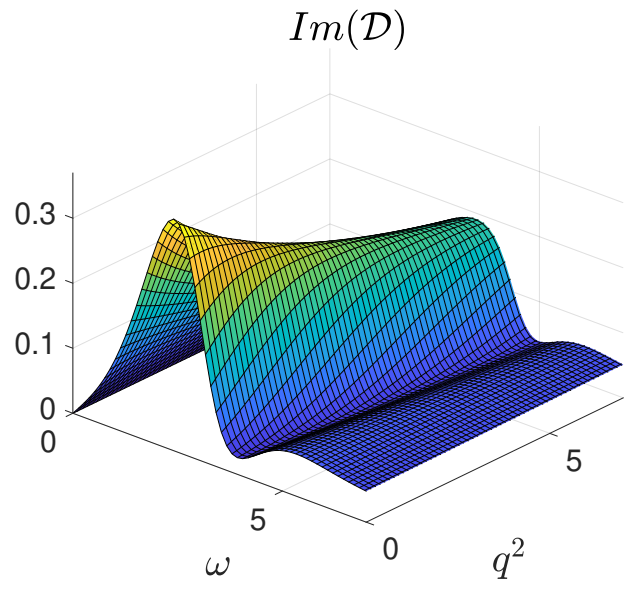

(b)

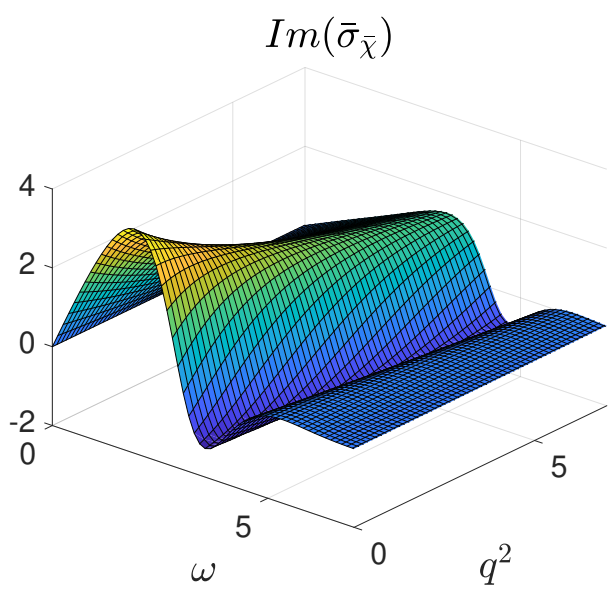

(d)

Figure 7. Diffusion function $\mathcal{D}$ and generalised CME/CSE conductivity $\bar{\sigma}_{\bar{\chi}}$ as functions of $\omega$ and $q^{2}$ when $\kappa \mathbf{B}=0.25$ and $\alpha=0$.

\subsection{The TCFs}

We are not able to solve the ODEs (4.21)-(4.26) analytically and thus resort to numerical techniques. The results are summarised in several plots below. First, we fix the magnetic field $\mathbf{B}$ and its relative angle $\alpha$ with respect to $\vec{q}$, and study the $\omega, q^{2}$-dependencies of the TCFs in (1.24), (1.25). Then, we examine the effects due to the magnetic field $\mathbf{B}$ and angle $\alpha$ variations.

In figures 7 and 8 , selecting representative values $\kappa \mathbf{B}=0.25$ and $\alpha=0$, we display 3D plots for all the TCFs in (1.24), (1.25) as functions of $\omega$ and $q^{2}$. All the TCFs exhibit a similar behavior: relatively weak dependence on the spatial momentum squared $q^{2}$, reflecting spatial quasi-locality of relevant transport processes; damped oscillations in the frequency $\omega$, towards asymptotic region where all the TCFs essentially vanish.

Figure 9 reveals the effect of variation of the angle $\alpha$ between $\overrightarrow{\mathbf{B}}$ and $\vec{q}$ on the TCFs. The results are normalised with respect to their values at $\alpha=\pi / 2$ denoted as $\mathcal{D}^{\perp}, \bar{\sigma}_{\bar{\chi}}^{\perp}$, 


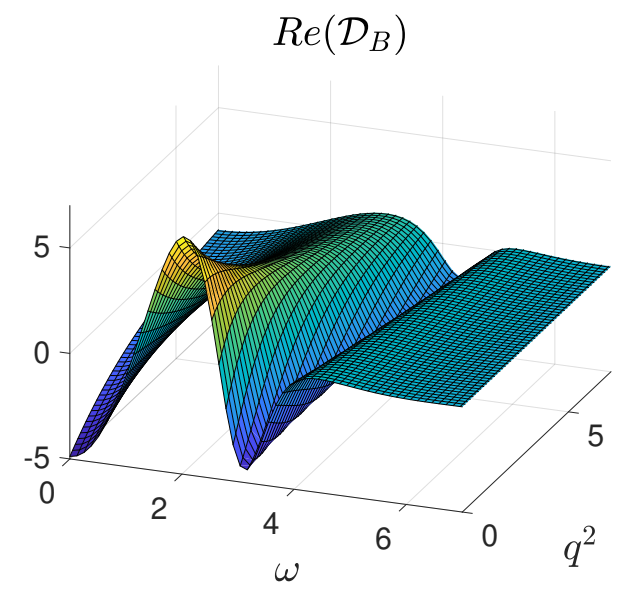

(a)

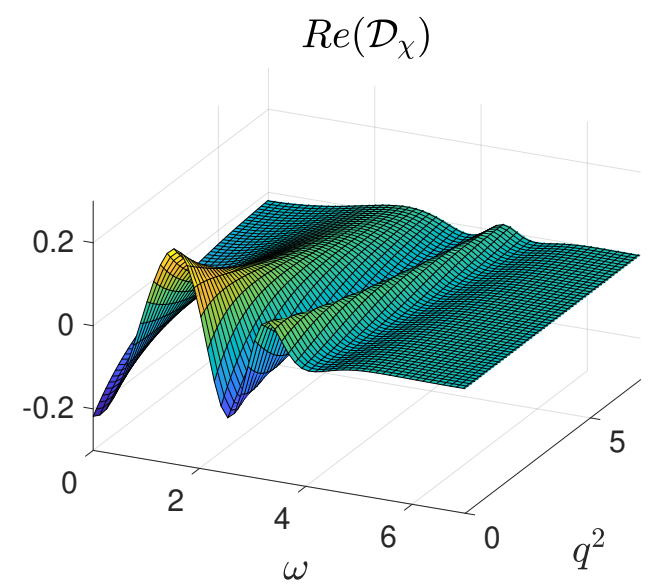

(c)

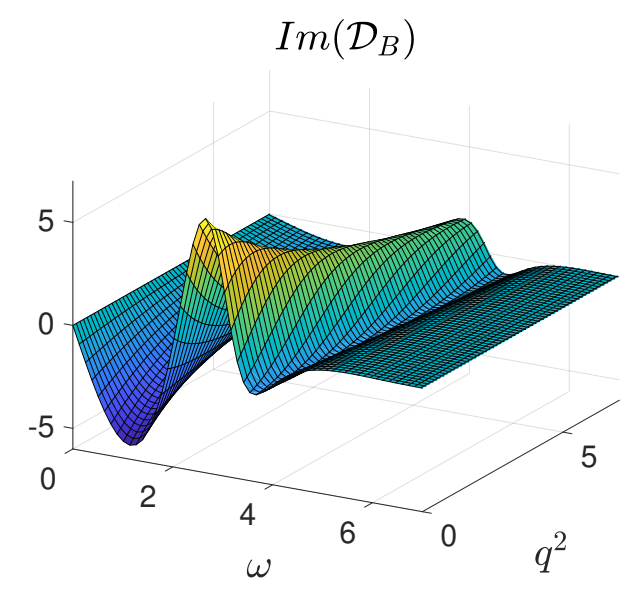

(b)

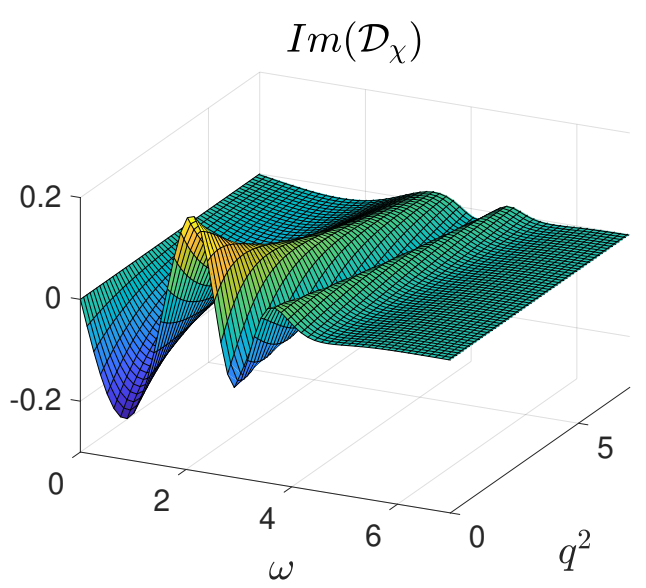

(d)

Figure 8. TCFs $\mathcal{D}_{B}$ and $\mathcal{D}_{\chi}$ as functions of $\omega$ and $q^{2}$ when $\kappa \mathbf{B}=0.25$ and $\alpha=0$.

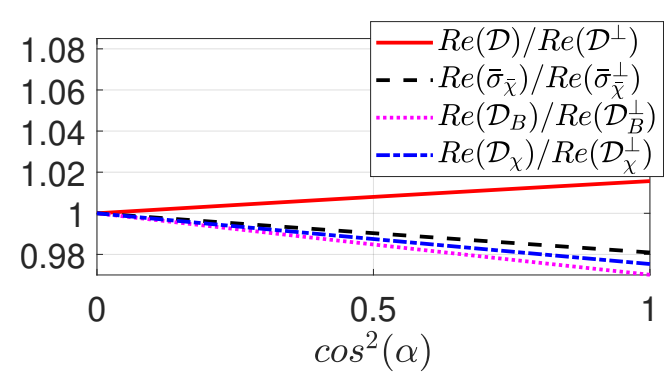

(a)

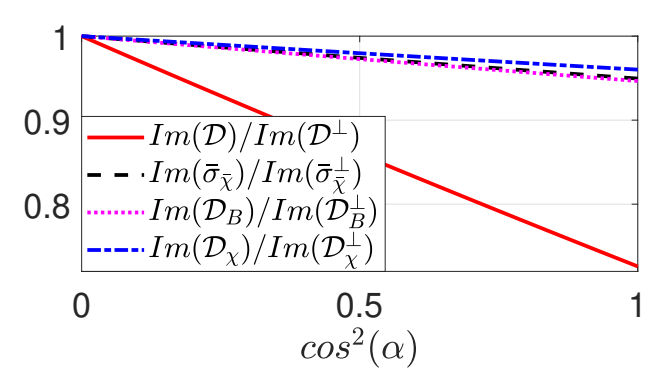

(b)

Figure 9. Normalised TCFs as functions of $\cos ^{2}(\alpha)$ when $\kappa \mathbf{B}=0.5$ and $\omega=q^{2}=0.1$.

$\mathcal{D}_{B}^{\perp}, \mathcal{D}_{\chi}^{\perp}$. In figure $9, \kappa \mathbf{B}=0.5$ and $\omega=q^{2}=0.1$. All the TCFs display a relatively mild dependence on $\cos ^{2}(\alpha)$. 


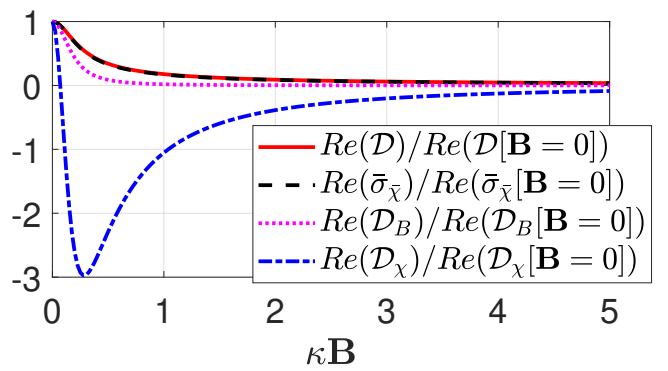

(a)

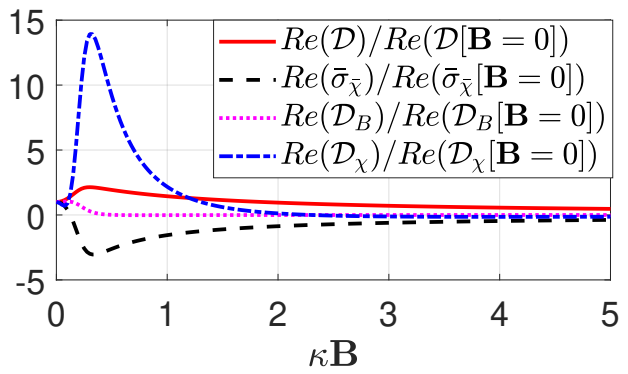

(c)

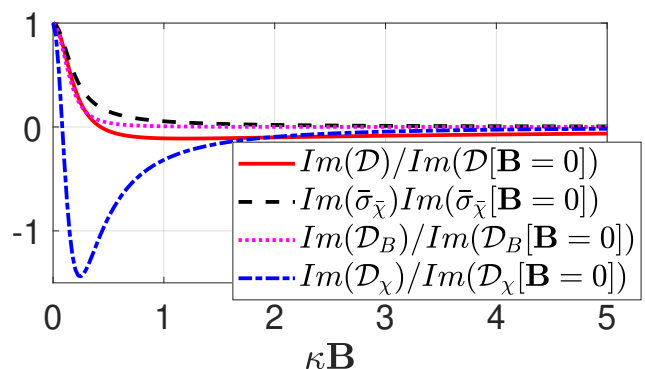

(b)

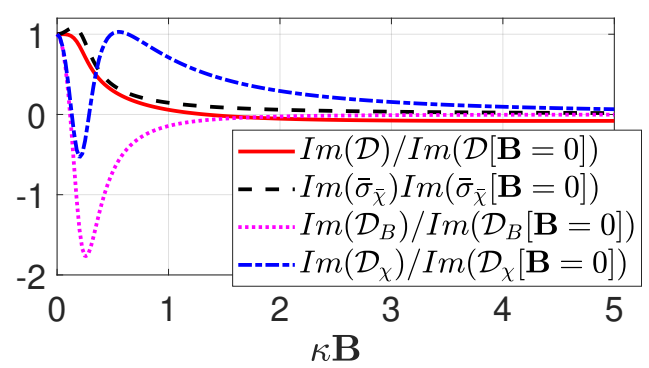

(d)

Figure 10. Real and imaginary parts of the TCFs as a function of $\kappa \mathbf{B}$ for (a), (b) $\omega=q^{2}=0.1$ and (c), (d) $\omega=q^{2}=2$.

In figure 10 we show the TCFs as functions of $\kappa \mathbf{B}$ while fixing all the rest of the parameters $\omega, q^{2}$ and $\alpha$. Since the latter effect is weak, we take $\alpha=0$ in the subsequent discussion. Regarding $\omega$ and $q^{2}$, we make two different choices to implement a comparative study: $\omega=q^{2}=0.1$ versus $\omega=q^{2}=2$. In figure 10, the TCFs are normalized with respect to their values when $\kappa \mathbf{B}=0$ with the same $\omega$ and $q^{2}$ values (which are obtained from our numerical results). All the TCFs are found to approach zero at large magnetic field. When $\omega, q^{2}$ are increased (i.e., with more non-hydrodynamic modes included), the asymptotic regime is shifted towards larger values of $\kappa \mathbf{B}$.

An interesting new phenomenon emerges at finite $\kappa \mathbf{B}$ : when the magnetic field is large enough, say $\kappa \mathbf{B} \gtrsim 0.5$, the TCFs develop singularity as functions of $\omega$, at real values of $\omega$ depending on $\kappa \mathbf{B}$ (see figure 11 as illustration). Moreover, all the TCFs (both real and imaginary parts) exhibit singularity at the same value of $\omega$. In figure $11, \operatorname{Re}\left[\bar{\sigma}_{\bar{\chi}}\right]$ is taken as an example demonstrating the phenomenon at $q=0$. If one continues to increase $\kappa \mathbf{B}$ and considers larger $\omega$, additional singularities emerge at larger values of $\omega$. The locations of these singularities $\omega_{n}$ are symmetric around the origin. These $\omega_{n}$ are presumably the quasinormal modes (QNM) in the bulk and here we find that they become real at some values of magnetic field. Within essentially the same holographic model, similar observation was made recently in [35]. 


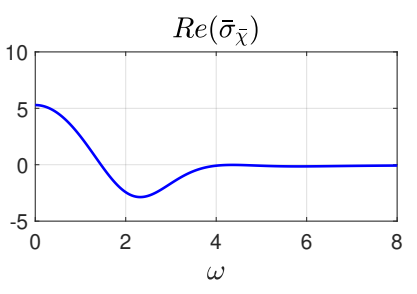

(a)

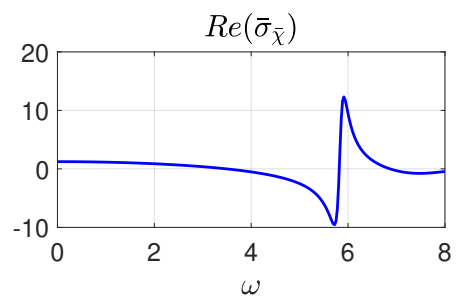

(d)

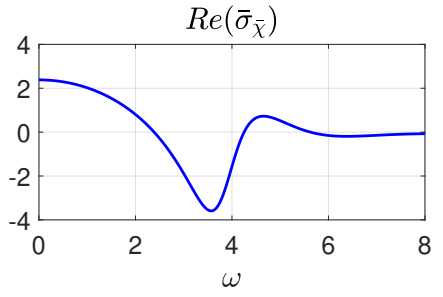

(b)

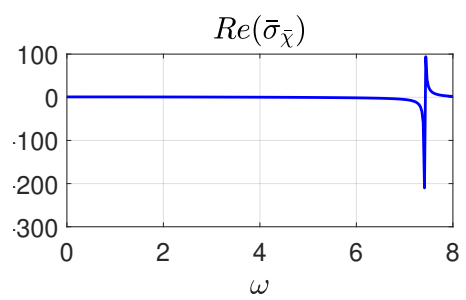

(e)

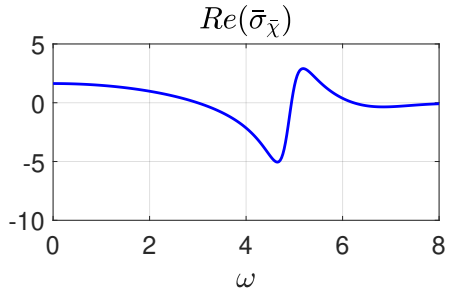

(c)

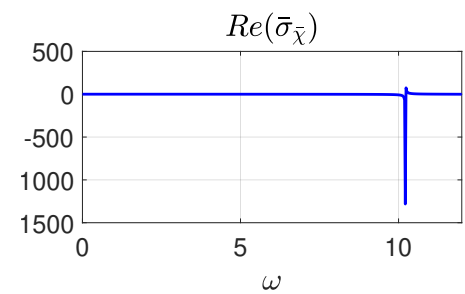

(f)

Figure 11. $\operatorname{Re}\left(\bar{\sigma}_{\bar{\chi}}\right)$ as a function of $\omega$ when $q=0$ and (a) $\kappa \mathbf{B}=0.1$, (b) $\kappa \mathbf{B}=0.4$, (c) $\kappa \mathbf{B}=0.6$, (d) $\kappa \mathbf{B}=0.8$, (e) $\kappa \mathbf{B}=1.2$, (f) $\kappa \mathbf{B}=2.2$.

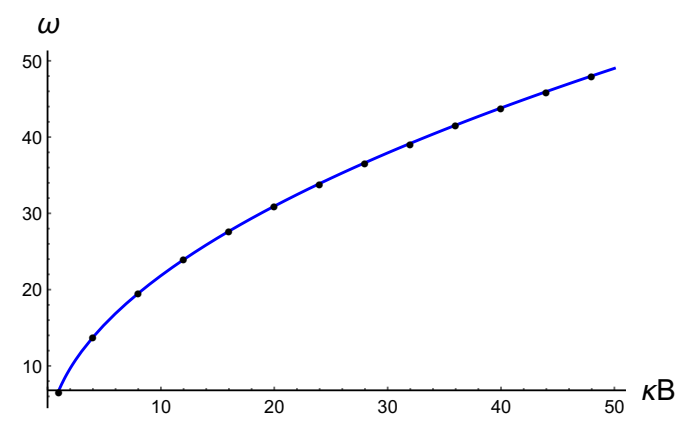

Figure 12. Location of the singularity as a function of $\kappa \mathbf{B}$ : numerical result (black dots) is best fitted by $\omega=-0.155157+6.95306 \sqrt{\kappa \mathbf{B}}$ (blue curve).

To conclude this part, we present the dependence of the lowest QNM mode on $\kappa \mathbf{B}$, see figure 12. The numerical results (black dots) are best fitted by:

$$
\omega=-0.155157+6.95306 \sqrt{\kappa \mathbf{B}}
$$

which is plotted as a continuous (blue) curve in figure 12. The mode with $\operatorname{Re}(\omega) \propto \sqrt{\kappa \mathbf{B}}$ while $\operatorname{Im}(\omega) \rightarrow 0$ is a manifestation of the Landau level behavior [32, 34]. Magnetic field dependence of the QNMs in a holographic model was first studied in $[39,40]$ but without any anomaly effects included.

\subsection{Non-dissipative CMW modes}

The original CMW is a dissipative wave at small momenta [30]. In [6], we asked the question if it can happen that, beyond the hydrodynamic limit, the dissipative (imaginary) part of the CMW vanishes. The answer is within the all order dispersion relation. We 
indeed found that the CMW possesses a discrete spectrum of non-dissipative modes when the magnetic field is larger than a critical value. Yet, the results of [6] were based on a "weak" magnetic field approximation. Below we reexamine the very same question, but now without any approximations involved.

The constitutive relations (1.24), (1.25), combined with the continuity equations (1.2), result in the exact CMW dispersion relation

$$
\omega= \pm\left(\bar{\sigma}_{\bar{\chi}}-q^{2} \mathcal{D}_{\chi}\right) \kappa \overrightarrow{\mathbf{B}} \cdot \vec{q}-i\left(q^{2} \mathcal{D}-\mathcal{D}_{B}(\kappa \overrightarrow{\mathbf{B}} \cdot \vec{q})^{2}\right)
$$

The last term is new compared to [6]. Furthermore, all the TCFs in (4.34) are functions of the magnetic field.

The procedure for finding a purely real solution was devised in [6]. To this goal, we first split the dispersion relation (1.26) into real and imaginary parts (assuming $\overrightarrow{\mathbf{B}} \| \vec{q}$ )

$$
\begin{aligned}
\phi_{R}\left(\omega, q^{2}, \kappa \mathbf{B}\right) & \equiv-\omega \pm \operatorname{Re}\left[\left(\bar{\sigma}_{\bar{\chi}}-q^{2} \mathcal{D}_{\chi}\right) \kappa q \mathbf{B}\right]+\operatorname{Im}\left[q^{2} \mathcal{D}-\mathcal{D}_{B}(\kappa q \mathbf{B})^{2}\right] \\
\phi_{I}\left(\omega, q^{2}, \kappa \mathbf{B}\right) & \equiv \pm \operatorname{Im}\left[\left(\bar{\sigma}_{\bar{\chi}}-q^{2} \mathcal{D}_{\chi}\right) \kappa q \mathbf{B}\right]-\operatorname{Re}\left[q^{2} \mathcal{D}-\mathcal{D}_{B}(\kappa q \mathbf{B})^{2}\right]
\end{aligned}
$$

Then we search for a real $\omega$ solution of two equations $\phi_{R}=0$ and $\phi_{I}=0$.

In figure 13, the functions $\phi_{R}$ and $\phi_{I}$ (with upper plus sign in (4.35)) are shown as contour plots in $\left(\omega, q^{2}\right)$ space for representative values of the magnetic field: (a) $\kappa \mathbf{B}=0.25$, (b) $\kappa \mathbf{B}=0.30$, (c) $\kappa \mathbf{B}=0.33$, and (d) $\kappa \mathbf{B}=0.5$. The dashed (blue) and solid (red) curves denote $\phi_{R}$ and $\phi_{I}$ respectively. The numbers indicated on the curves are the values of these functions along the curves. Therefore, any crossing point of $\phi_{R}=0$ (bold dashed blue curve) and $\phi_{I}=0$ (bold solid red curve) implies that both functions vanish simultaneously. This is the desired solution.

In [6], such a crossing occurred at a "single point" in the $\left(\omega, q^{2}\right)$ space, for each $\kappa \mathbf{B} \geq 0.33$, similarly to the case shown in figure 13a. However, beyond the weak field approximation, new branches emerge as demonstrated in figures 13b), (13c and 13d: the contours $\phi_{R}=0$ and $\phi_{I}=0$ coincide within a "continuous interval" in the $\left(\omega, q^{2}\right)$ space, starting from a large enough $\kappa \mathbf{B}$. This implies a continuum of non-dissipative CMW modes. In figure 13d, it seems like the contours $\phi_{R}=0$ and $\phi_{I}=0$ coincide completely, though the figure is somewhat misleading. Indeed, by zooming in $\left(\omega, q^{2}\right)$ space, we observe that the solution exists only on piece-wise intervals in the $\left(\omega, q^{2}\right)$ space. It is also worth adding that there are additional solution branches at larger $\omega$, which we do not display here.

Finally, the results above were obtained for non-dissipative CMW propagating along the magnetic field direction. One can ask the question if similar wave could propagate at an angle $\alpha$ with respect to the magnetic field, or even orthogonal to it. We briefly report that changing $\alpha$ from $\vec{q} \| \overrightarrow{\mathbf{B}}$ to $\vec{q} \perp \overrightarrow{\mathbf{B}}$ leads to separation of $\phi_{R}=0$ and $\phi_{I}=0$ contours. Non-dissipative solutions disappear at a critical value of $\alpha=\alpha_{c}[\kappa \mathbf{B}]$ (e.g. $\alpha_{c} \simeq \pi / 6$ for $\kappa \mathbf{B}=0.33)$. 


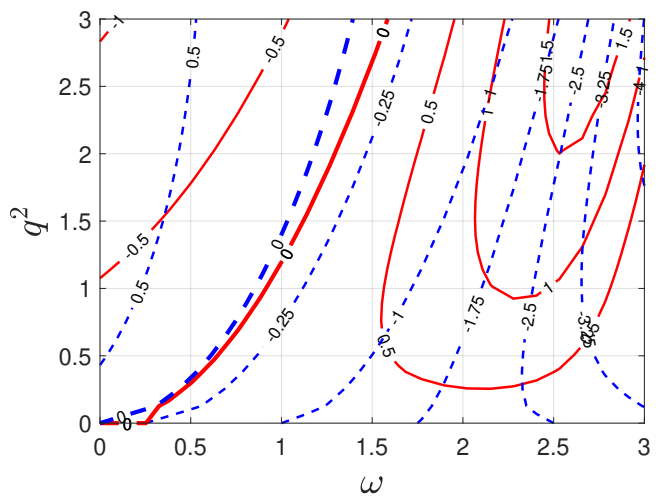

(a)

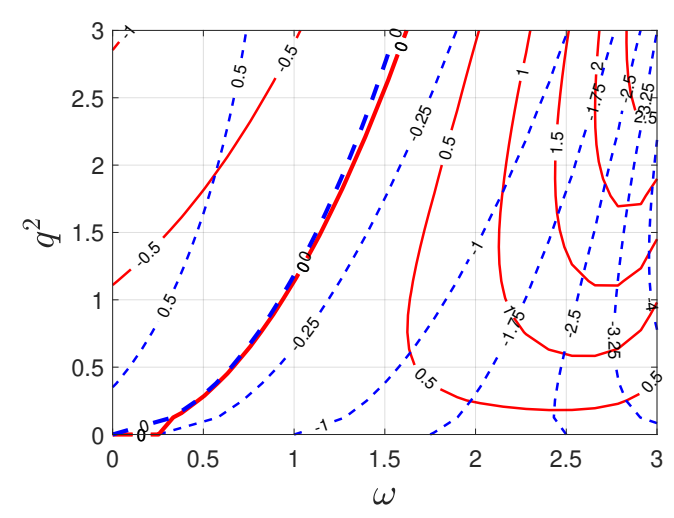

(c)

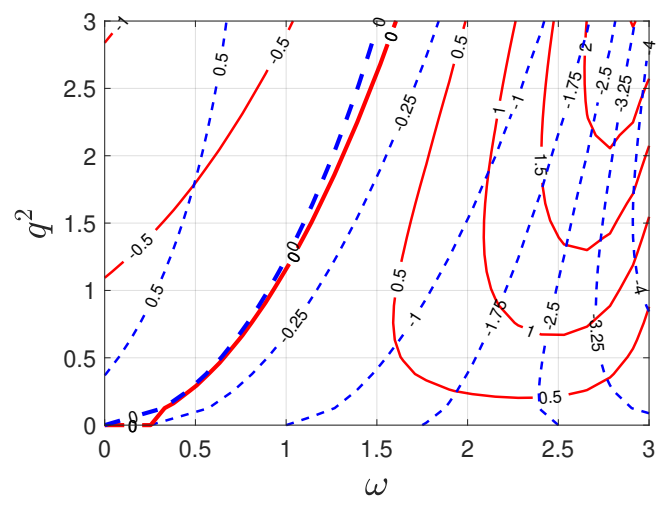

(b)

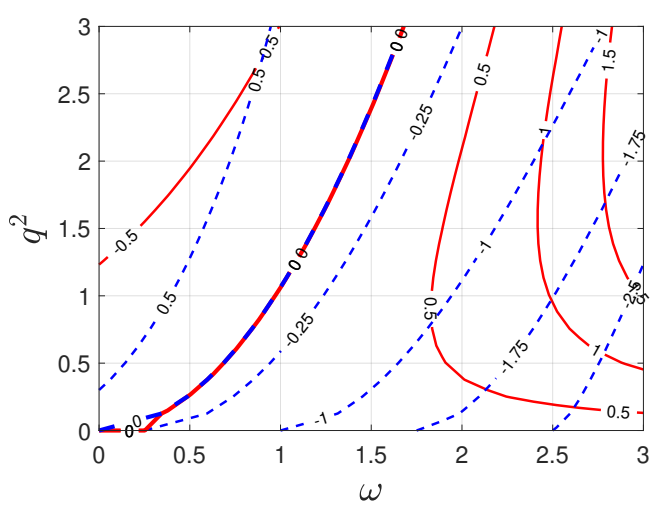

(d)

Figure 13. Contour plots for the functions $\phi_{R}$ (blue dashed) and $\phi_{I}$ (red solid) at (a) $\kappa \mathbf{B}=0.25$, (b) $\kappa \mathbf{B}=0.30$, (c) $\kappa \mathbf{B}=0.33$, (d) $\kappa \mathbf{B}=0.5$.

\section{Concluding remarks}

In this work we focused on the influence of strong background $\mathrm{e} / \mathrm{m}$ fields on the chiral anomaly-induced transport phenomena for a holographically defined thermal plasma. The constitutive relations for the vector and axial currents $\vec{J}, \vec{J}_{5}$ were evaluated within two complementary approximation schemes: fixed order gradient expansion (up to the first order) and the all-order gradient resummation (linear in the charge densities $\rho, \rho_{5}$ ). A summary of all the results could be found in the introductory section. The main highlights of our study are:

- There are three types of gapless modes propagating in the chiral plasma: the chiral magnetic wave (CMW) [30], the chiral electric wave (CEW) [24] and the chiral Hall density wave (CHDW) [5, 6], which could be searched experimentally in heavy ion collisions or, more likely, in condensed matter experiments where the external fields are under better control.

- While most of the transport coefficients are found to be suppressed by the external fields and vanish at asymptotically strong fields, the Ohmic conductivity gets 
enhanced in parallel electrical and magnetic fields, which is an experimentally interesting phenomenon to be searched for.

- Some anomaly-induced transport phenomena display noticeable dependence on the relative angle between the external fields $\overrightarrow{\mathbf{E}}$ and $\overrightarrow{\mathbf{B}}$. This sensitivity could be used in real experiments to zoom into one or another anomaly-induced phenomena.

- When $\overrightarrow{\mathbf{E}}=0$, the all-order resummed constitutive relations (1.24), (1.25) are parameterised by four independent TCFs, which are functions of $\omega, \vec{q}$ and $\overrightarrow{\mathbf{B}}$. Intriguingly, these TCFs are found to show a common singularity at certain value of real $\omega$ when $\kappa \mathbf{B}$ is strong enough. Moreover, this singularity is identified as QNM frequency and obey Landau level behavior (4.33) as a function of $\kappa \mathbf{B}$.

- In [6] we discovered a discrete set of entirely non-dissipative and thus long-lived CMW modes emerging in a weak magnetic field. Present work examined CMW exactly without the weak field approximation. We found that, depending on the magnetic field, the discrete set extends into several continuous intervals in the $\left(\omega, q^{2}\right)$ space. This effect should have a clear experimental signature worth exploring.

Real experiments involving chiral plasma, such as the one produced in heavy ion collisions or the primordial plasma in early Universe, involve strong dynamical fields with non-homogeneous profiles. While the study reported above was limited to constant background fields, it revealed important new anomaly-induced phenomena and serves as a step towards development of a self-consistent chiral MHD. Any dynamical simulations of the latter are beyond the scope of this paper.

\section{Acknowledgments}

We would like to thank Umut Gürsoy and Shu Lin for useful discussions. YB would like to thank the hospitality of the Department of Physics at Ben-Gurion University of the Negev where this work was initiated. YB was supported by the Fundamental Research Funds for the Central Universities under grant No.122050205032 and the Natural Science Foundation of China (NSFC) under the grant No.11705037. TD and ML were supported by the Israeli Science Foundation (ISF) grant \#1635/16 and the BSF grant \#2014707.

Open Access. This article is distributed under the terms of the Creative Commons Attribution License (CC-BY 4.0), which permits any use, distribution and reproduction in any medium, provided the original author(s) and source are credited.

\section{References}

[1] H.-U. Yee, Holographic Chiral Magnetic Conductivity, JHEP 11 (2009) 085 [arXiv: 0908.4189] [INSPIRE].

[2] A. Gynther, K. Landsteiner, F. Pena-Benitez and A. Rebhan, Holographic Anomalous Conductivities and the Chiral Magnetic Effect, JHEP 02 (2011) 110 [arXiv:1005.2587] [INSPIRE]. 
[3] Y. Bu, M. Lublinsky and A. Sharon, Anomalous transport from holography: Part I, JHEP 11 (2016) 093 [arXiv: 1608.08595] [INSPIRE].

[4] Y. Bu, M. Lublinsky and A. Sharon, Anomalous transport from holography: Part II, Eur. Phys. J. C 77 (2017) 194 [arXiv: 1609.09054] [INSPIRE].

[5] Y. Bu, T. Demircik and M. Lublinsky, Nonlinear chiral transport from holography, JHEP 01 (2019) 078 [arXiv: 1807. 08467] [inSPIRE].

[6] Y. Bu, T. Demircik and M. Lublinsky, Gradient resummation for nonlinear chiral transport: an insight from holography, Eur. Phys. J. C 79 (2019) 54 [arXiv:1807.11908] [INSPIRE].

[7] S. Lin and H.-U. Yee, Out-of-Equilibrium Chiral Magnetic Effect at Strong Coupling, Phys. Rev. D 88 (2013) 025030 [arXiv:1305.3949] [INSPIRE].

[8] A. Boyarsky, J. Fröhlich and O. Ruchayskiy, Self-consistent evolution of magnetic fields and chiral asymmetry in the early Universe, Phys. Rev. Lett. 108 (2012) 031301 [arXiv:1109.3350] [INSPIRE].

[9] A. Boyarsky, J. Fröhlich and O. Ruchayskiy, Magnetohydrodynamics of Chiral Relativistic Fluids, Phys. Rev. D 92 (2015) 043004 [arXiv:1504.04854] [INSPIRE].

[10] C. Manuel and J.M. Torres-Rincon, Dynamical evolution of the chiral magnetic effect: Applications to the quark-gluon plasma, Phys. Rev. D 92 (2015) 074018 [arXiv:1501. 07608] [INSPIRE].

[11] X.-G. Huang, Electromagnetic fields and anomalous transports in heavy-ion collisions - A pedagogical review, Rept. Prog. Phys. 79 (2016) 076302 [arXiv:1509. 04073] [INSPIRE].

[12] D.E. Kharzeev, J. Liao, S.A. Voloshin and G. Wang, Chiral magnetic and vortical effects in high-energy nuclear collisions - A status report, Prog. Part. Nucl. Phys. 88 (2016) 1 [arXiv: 1511.04050] [INSPIRE].

[13] V. Koch et al., Status of the chiral magnetic effect and collisions of isobars, Chin. Phys. C 41 (2017) 072001 [arXiv: 1608.00982] [INSPIRE].

[14] Y. Bu and M. Lublinsky, Linearly resummed hydrodynamics in a weakly curved spacetime, JHEP 04 (2015) 136 [arXiv: 1502.08044] [INSPIRE].

[15] Y. Bu, M. Lublinsky and A. Sharon, U(1) current from the AdS/CFT: diffusion, conductivity and causality, JHEP 04 (2016) 136 [arXiv:1511.08789] [INSPIRE].

[16] M. Lublinsky and E. Shuryak, How much entropy is produced in strongly coupled quark-gluon Plasma (sQGP) by dissipative effects?, Phys. Rev. C 76 (2007) 021901 [arXiv:0704.1647] [INSPIRE].

[17] M. Lublinsky and E. Shuryak, Improved Hydrodynamics from the AdS/CFT, Phys. Rev. D 80 (2009) 065026 [arXiv:0905.4069] [INSPIRE].

[18] Y. Bu and M. Lublinsky, All order linearized hydrodynamics from fluid-gravity correspondence, Phys. Rev. D 90 (2014) 086003 [arXiv:1406.7222] [INSPIRE].

[19] Y. Bu and M. Lublinsky, Linearized fluid/gravity correspondence: from shear viscosity to all order hydrodynamics, JHEP 11 (2014) 064 [arXiv:1409.3095] [INSPIRE].

[20] Y. Bu, M. Lublinsky and A. Sharon, Hydrodynamics dual to Einstein-Gauss-Bonnet gravity: all-order gradient resummation, JHEP 06 (2015) 162 [arXiv:1504.01370] [INSPIRE].

[21] A. Vilenkin, Equilibrium parity-violating current in a magnetic field, Phys. Rev. D 22 (1980) 3080. 
[22] K. Fukushima, D.E. Kharzeev and H.J. Warringa, The Chiral Magnetic Effect, Phys. Rev. D 78 (2008) 074033 [arXiv: 0808.3382] [INSPIRE].

[23] D.T. Son and P. Surowka, Hydrodynamics with Triangle Anomalies, Phys. Rev. Lett. 103 (2009) 191601 [arXiv:0906 . 5044] [INSPIRE].

[24] S. Pu, S.-Y. Wu and D.-L. Yang, Chiral Hall Effect and Chiral Electric Waves, Phys. Rev. D 91 (2015) 025011 [arXiv: 1407.3168] [INSPIRE].

[25] D.T. Son and A.R. Zhitnitsky, Quantum anomalies in dense matter, Phys. Rev. D 70 (2004) 074018 [hep-ph/0405216] [INSPIRE].

[26] M.A. Metlitski and A.R. Zhitnitsky, Anomalous axion interactions and topological currents in dense matter, Phys. Rev. D 72 (2005) 045011 [hep-ph/0505072] [INSPIRE].

[27] X.-G. Huang and J. Liao, Axial Current Generation from Electric Field: Chiral Electric Separation Effect, Phys. Rev. Lett. 110 (2013) 232302 [arXiv:1303.7192] [InSPIRE].

[28] D.O. Rybalka, E.V. Gorbar and I.A. Shovkovy, Hydrodynamic modes in a magnetized chiral plasma with vorticity, Phys. Rev. D 99 (2019) 016017 [arXiv: 1807.07608] [InSPIRE].

[29] I.A. Shovkovy, D.O. Rybalka and E.V. Gorbar, The overdamped chiral magnetic wave, in 13th Conference on Quark Confinement and the Hadron Spectrum (Confinement XIII), Maynooth Ireland (2018) [arXiv: 1811.10635] [INSPIRE].

[30] D.E. Kharzeev and H.-U. Yee, Chiral Magnetic Wave, Phys. Rev. D 83 (2011) 085007 [arXiv: 1012.6026] [INSPIRE].

[31] K. Landsteiner, Y. Liu and Y.-W. Sun, Negative magnetoresistivity in chiral fluids and holography, JHEP 03 (2015) 127 [arXiv:1410.6399] [INSPIRE].

[32] M. Ammon, S. Grieninger, A. Jimenez-Alba, R.P. Macedo and L. Melgar, Holographic quenches and anomalous transport, JHEP 09 (2016) 131 [arXiv:1607.06817] [INSPIRE].

[33] E.V. Gorbar, I.A. Shovkovy, S. Vilchinskii, I. Rudenok, A. Boyarsky and O. Ruchayskiy, Anomalous Maxwell equations for inhomogeneous chiral plasma, Phys. Rev. D 93 (2016) 105028 [arXiv: 1603.03442] [INSPIRE].

[34] M. Ammon, M. Kaminski, R. Koirala, J. Leiber and J. Wu, Quasinormal modes of charged magnetic black branes \&̇amp; chiral magnetic transport, JHEP 04 (2017) 067 [arXiv: 1701.05565] [INSPIRE].

[35] M. Haack, D. Sarkar and A. Yarom, Probing anomalous driving, JHEP 04 (2019) 034 [arXiv: 1812.08210] [INSPIRE].

[36] G.T. Horowitz, N. Iqbal and J.E. Santos, Simple holographic model of nonlinear conductivity, Phys. Rev. D 88 (2013) 126002 [arXiv:1309.5088] [INSPIRE].

[37] H.B. Zeng, Y. Tian, Z.Y. Fan and C.-M. Chen, Nonlinear Transport in a Two Dimensional Holographic Superconductor, Phys. Rev. D 93 (2016) 121901 [arXiv:1604.08422] [INSPIRE].

[38] H.-B. Zeng, Y. Tian, Z. Fan and C.-M. Chen, Nonlinear Conductivity of a Holographic Superconductor Under Constant Electric Field, Phys. Rev. D 95 (2017) 046014 [arXiv: 1611.06798] [INSPIRE].

[39] S. Janiszewski and M. Kaminski, Quasinormal modes of magnetic and electric black branes versus far from equilibrium anisotropic fluids, Phys. Rev. D 93 (2016) 025006 [arXiv: 1508.06993] [INSPIRE].

[40] T. Demircik and U. Gürsoy, Holographic equilibration in confining gauge theories under external magnetic fields, Nucl. Phys. B 919 (2017) 384 [arXiv:1605.08118] [INSPIRE]. 